\title{
Ensuring fair access in IEEE 802.11p-based vehicle-to-infrastructure networks
}

\author{
Vettath Pathayapurayil Harigovindan*, Anchare V Babu and Lillykutty Jacob
}

\begin{abstract}
IEEE $802.11 \mathrm{p}$ is an approved amendment to the IEEE 802.11 standard to facilitate wireless access in vehicular environments (WAVE). In this article, we present an analytical model to evaluate the impact of vehicle mobility on the saturation throughput of IEEE 802.11p-based vehicle-to-infrastructure (V2I) networks. The throughput model is then used to investigate an unfairness problem that exists in such networks among vehicles with different mobility characteristics. Assuming a saturated network, if all the vehicles in the network use the same MAC parameters, IEEE 802.11 p MAC protocol provides equal transmission opportunity for all of them, provided they have equal residence time in the coverage area of a road side unit (RSU). When vehicles have different mobility characteristics (e.g., extremely high and low speeds), they do not have similar chances of channel access. A vehicle moving with higher velocity has less chance to communicate with its RSU, as compared to a slow moving vehicle, due to its short residence time in the coverage area of RSU. Accordingly, the data transfer of a higher velocity vehicle gets degraded significantly, as compared to that of the vehicle with lower velocity, resulting in unfairness among them. In this article, our aim is to address this unfairness problem that exists among vehicles of different velocities in V2I networks. Analytical expressions are derived for optimal minimum CW (CW min $)$ required to ensure fairness, in the sense of equal chance of communicating with RSU, among competing vehicles of different mean velocities in the network. Analytical results are validated using extensive simulations.
\end{abstract}

Keywords: IEEE 802.11p, fairness, residence time, saturation throughput, vehicle-to-infrastructure networks

\section{Introduction}

Vehicular ad-hoc network (VANET) is an emerging wireless network in which vehicles constitute the mobile nodes in the network. Such networks are aimed at providing support for road safety, traffic management, and comfort applications by enabling vehicle-to-vehicle (V2V) or vehicle-to-infrastructure (V2I) communications [1,2]. The emerging technology for VANETs is the dedicated short range communications (DSRC), for which the Federal Communications Commission in the United States has allocated $75 \mathrm{MHz}$ of spectrum between 5850 and 5925 MHz. The DSRC is based on IEEE 802.11 technology and is proceeding towards standardization under the standard IEEE 802.11p, whereas the entire communication stack is being standardized by the IEEE 1609 working group under the name wireless access in vehicular environments (WAVE). The overall WAVE architecture includes IEEE

\footnotetext{
* Correspondence: harigovindan_pec10@nitc.ac.in

Department of Electronics and Communication Engineering, National Institute of Technology Calicut, Calicut, 673601, India
}

standards 1609.1 to 1609.4 (for resource management, security architecture, networking service, and multi-channel operation, respectively) and IEEE 802.11p (MAC and PHY standard). IEEE 802.11p uses essentially the same PHY defined for 802.11 a but operates in a $10 \mathrm{MHz}$ wide channel instead of $20 \mathrm{MHz}$. The goal of 802.11p standard is to provide V2V and V2I communications over the dedicated $5.9 \mathrm{GHz}$ licensed frequency band and supports data rates of 3-27 Mbps (3, 4.5, 6, 9, 12, 18, 24, and $27 \mathrm{Mbps})$ $[3,4]$.

Future intelligent transportation systems (ITS) will necessitate wireless V2I communications. Besides the delivery of infotainment services, the role of typical V2I systems will include the provisioning of safety related, real-time, local, and situation-based services, such as speed limit information, safe distance warning, lane keeping support, intersection safety, traffic jam, and accident warning, etc. All these services aim to prevent accidents by providing timely information directly to the car and/ or to the driver. The main technical challenges for

\section{Springer}


communication in V2I and V2V networks are the very high mobility of the nodes, highly dynamic topology, high variability in node density, and very short duration of communication [1-3]. The IEEE 802.11p uses the enhanced distributed channel access (EDCA) medium access control (MAC) sublayer protocol based on distributed coordination function (DCF) [4]. DCF, which is based on Carrier Sense Multiple Access with Collision Avoidance (CSMA/CA), was originally developed for WLANs [5]. Under DCF, all stations compete for access by using CSMA/CA protocol; they sense the channel before transmitting, and if the medium is found idle for a minimum time equal to DIFS, the packet will be transmitted. Otherwise, the station enters backoff and randomly sets its backoff counter within the range of its Contention Window $(\mathrm{CW})$. The random discrete back off time is selected from the range [0,CW-1]. To transmit packets after DIFS, node first decrements the backoff time counter and transmit only if the backoff counter is 0 . The countdown of backoff counter is frozen once the channel becomes busy due to other node transmission, and resumes when the channel is idle for another DIFS. The size of CW depends on the history of transmissions. At the first transmission attempt, it is set to a predefined value $C W_{\min }$, the minimum $C W$. Upon each unsuccessful transmissions, it is updated to $2^{s} C W_{\min }$ until it reaches a maximum value $C W_{\max }$. Here $s$ is called backoff stage. Each station maintains a retry counter that indicates the number of retransmission attempts of a data packet. More details of DCF can be found in [5]. The EDCA mechanism assigns four different priority classes for incoming packets at each node which are called Access Categories (AC). Each AC has its own channel access function when compared with 802.11 DCF in which all packets exploit the same access function to acquire the channel. Different access functions for different categories mean assigning different delay times, different minimum contention windows, and different number of back-off stages for each type of service [6].

The DCF protocol was originally developed for low mobility networks such as WLANs and recent studies have shown that it does not operate efficiently for a high mobility communication scenario such as vehicular networks. In static networks, the performance of the IEEE 802.11 DCF depends on network parameters such as the number of communicating nodes, type of data traffic, backoff procedure, packet size, data rates of different nodes, etc. [7-11]. In vehicular communication networks, the performance of DCF protocol is also affected by other factors such as vehicle density and node mobility [12-16]. Node mobility can be characterized by node position, speed, and acceleration, direction of movement, potential communication duration, and potential number of communication neighbors. All these factors are highly dynamic in V2I networks, and difficult to predict especially in an extreme mobility environment.

The problem of unfairness due to vehicles having different velocities has been reported in [17] for the V2I communication scenario, involving fixed RSU. The standard IEEE 802.11p does not take into consideration, the resident time of nodes within the coverage of each RSU. Vehicles having different velocities have different resident times in the coverage area of an RSU. Assuming a saturated network, if all the vehicles in the network use the same MAC parameters, DCF protocol provides equal transmission opportunity for all of them. When vehicles have different mobility characteristics (e.g., extremely high and low speeds), they do not have similar chances of communication with RSU due to the different resident times and, therefore, a fairness problem exists. A fast moving vehicle has less chance to communicate with its RSU and consequently less amount of data transferred as compared to a slow moving vehicle. This problem occurs for each area covered by an RSU. Therefore, the amount of data transferred at each area (useful for next areas) is not equal. The contiguous areas covered by different RSUs and handoffs between them do not solve this problem. Since emergency information are more vital for faster vehicles, the above problem has more degrading effect on the efficiency of VANETs. In this article, our aim is to resolve this unfairness problem by adjusting the transmission probability of each vehicle according to its speed, through changing the minimum contention window size. In this way, the amount of successfully transmitted data of all nodes are made equal regardless of their velocities, while in the coverage area of an RSU. Using Jain's fairness index, we show how fairness in the sense of equal chance of communicating with RSU can be achieved by a judicious choice of minimum $\mathrm{CW}$ for the competing vehicles of distinct velocities in the network. The impact of these choices on throughput are also presented. The analytical findings are verified with extensive simulation studies.

The major contributions of this article are as follows:

- A modified analytical model for DCF is developed for V2I networks for computing individual vehicle throughput when vehicles are moving at very high velocities. The problem of unfairness that arises due to different vehicle velocities is investigated using this model. Analytical expressions for optimal minimum CW parameters to achieve the desired fairness objectives are derived.

- Extensive analytical and simulation results are provided to support the claims.

The rest of this article is organized as follows. Section 2 presents related study. In Section 3, we present an analytical model to compute the saturation throughput 
of a V2I network. In Section 4, we discuss how fairness in data transfer to RSU for individual node can be ensured. The analytical and simulation results are presented in Section 5. The article is concluded in Section 6.

\section{Related work}

The performance of DCF has been extensively studied in the literature [6-11]. Furthermore, an extensive body of research has been devoted to the performance evaluation of IEEE $802.11 \mathrm{p}$ standard [18-25]. A performance evaluation of IEEE 802.11p WAVE standard, considering collision probability, throughput and delay, is presented in [18] using simulations and analytical means. Studies show that WAVE can prioritize messages; however, in dense and high load scenarios the throughput decreases and the delay increases significantly. Authors of $[12,13]$ propose analytical model to evaluate performance and reliability of IEEE 802.11a-based V2V safety-related broadcast services in DSRC system on highway. In [14], simulation results of IEEE 802.11p MAC protocol are presented for the V2I scenario. The authors show that the specified MAC parameters for this protocol can lead to undesired throughput performance under dense and dynamic conditions. Authors of [15] propose a simple but accurate analytical model to evaluate the throughput performance of DCF in the high speed V2I communications. They show that with node velocity increasing, throughput of DCF decreases monotonically due to mismatch between $\mathrm{CW}$ and mobility. Using a $p$-persistent CSMA based model, they analyze the performance when different $p$ parameter values are assigned to nodes with different data rates (determined by the different distances from the RSU). In [19], the same authors used a 3D Markov chain to evaluate the throughput of DCF in the drive-thru internet scenario. Their proposals for protocol enhancement are $(i) C W_{\min }$ should be adapted to the data rates of the vehicles (according to their distances from RSU) and also to the vehicle velocity; and (ii) the maximum backoff stage should be kept small $(m=1)$ to mitigate the impact of mobility. In [16], authors propose an analytic model to evaluate the DSRC-based inter-vehicle communication. The impacts of the channel access parameters associated with different services including arbitration inter-frame space (AIFS) and contention window $(\mathrm{CW})$ are investigated. In [20], Suthaputchakun and Ganz study the use of IEEE 802.11e for priority based safety messaging for V2V in VANETs. Analytical model for DSRC network that uses the IEEE 802.11 DCF MAC protocol is developed in [21]. In [22,23], Tan et al. derive analytical models to characterize the average and the distribution of the number of bytes downloaded by a vehicle by the end of its sojourn through an AP's coverage range, in the presence of contention by other vehicles. Authors of [24], propose a new vehicular channel access scheme to compromise the trade-off between system throughput and throughput fairness in V2I communication scenario.

The problem of unfairness due to vehicles having different velocities has been explained for a V2I scenario in [17] and for a V2V scenario in [25]. Karamad and Ashtiani [17] present an analysis, in which the network that spans the coverage area of RSU is modeled as an $M / G / \infty$ queue. Customers in this queue are the batches of vehicles entering the network, with vehicles in a batch having the same speed. They divide the batches of vehicles according to their speed into $P$ classes. For class $i$, the service time $T_{i}$ in the $M / G / \infty$ queuing model is the residence time in the coverage area of RSU. Using this model, they obtain an expression for the saturation throughput. They also approximate the number of packets transmitted by a node during its residence time by a poisson random variable. Using these approximations and results from Bianchi's analysis [7], they derive an approximation for the optimal $C W_{\min }$ for fair access. In [25], Alasmary and Zhuang propose two dynamic CW based mechanisms to alleviate the performance degradation caused by vehicle mobility in V2V networks. But the article does not describe the exact procedure for the selection of optimal $\mathrm{CW}$ value to achieve the objectives. In this article, we present a simple yet accurate analytical model for DCF in high mobility scenario of V2I networks, and use this model to analyze the problem of unfairness that arises due to different vehicle velocities. We derive expressions for optimal minimum CW for vehicles with different mean velocities to achieve the desired fairness objectives. Extensive studies of the impact of parameters such as vehicle arrival rate, vehicle density, mean vehicle speed, traffic jam density, and number of nodes, on the amount of data transmitted by each vehicle during its sojourn time, are conducted.

\section{Analytical model for computing saturation throughput in V2I network}

Our system model is similar to that of $[15,19]$. Consider the vehicle to infrastructure (V2I) scenario, as shown in Figure 1, with vehicles connecting to intermittent and serial RSUs along the road. Assume that each node (vehicle) has always a frame ready for transmission (i.e., saturation assumption). Further, we assume perfect channel conditions (i.e., no transmission errors), and ignore the effect of hidden and exposed terminals. Such assumptions are typically used for the performance analysis of MAC layer throughput of wireless networks $[6-11,15,19]$.

Generally in V2I like application specific networks, service providers will not pursue full coverage because of the high deployment and maintenance costs, which in 


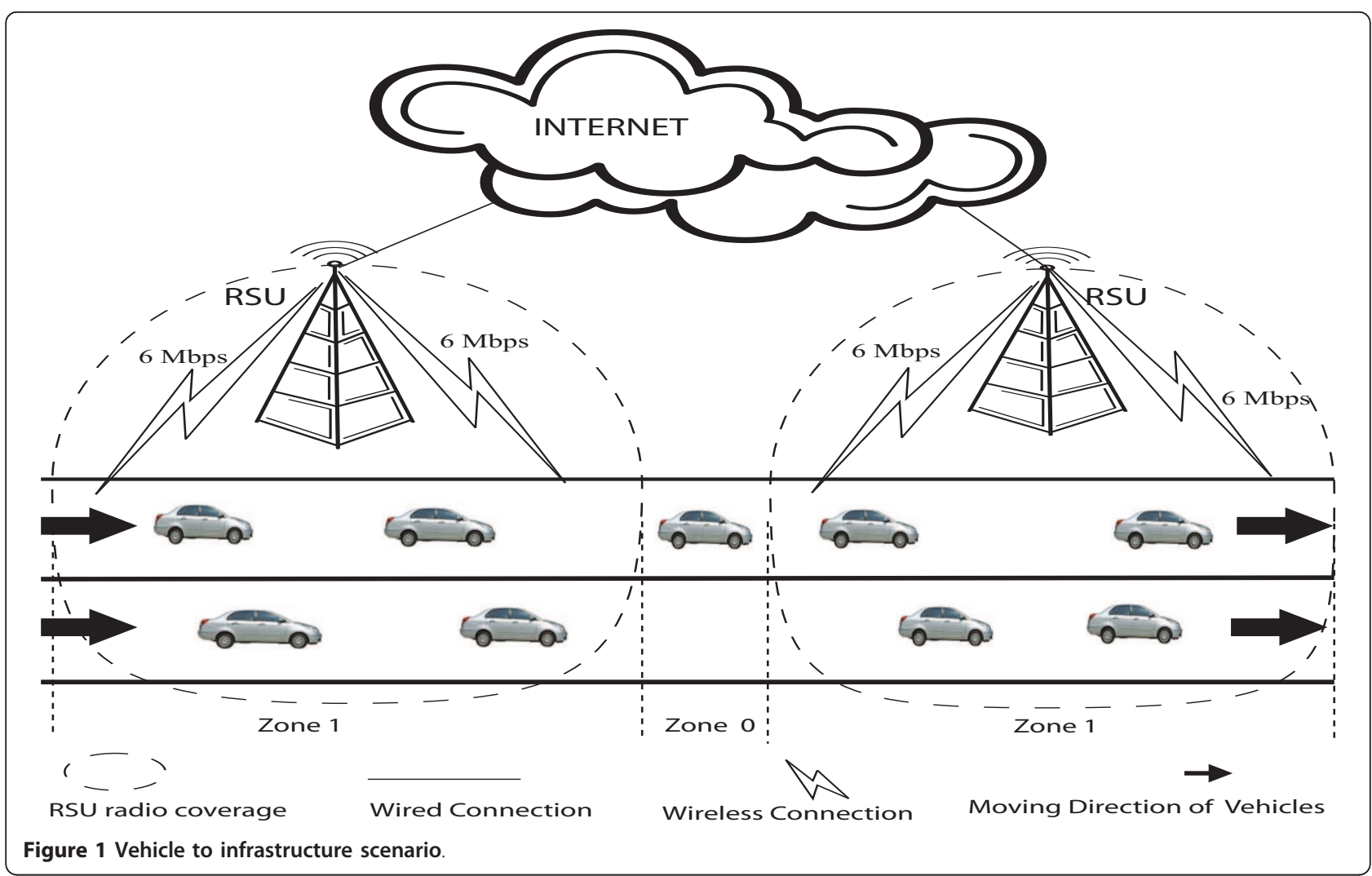

turn, makes non-coverage areas in the network. Even if they provide full coverage with contiguous areas covered by different RSUs and hand offs between them, some emergency information (e.g., status of traffic load, probable crashes occurred in the next road, etc.) must be communicated at each area. Since we are interested in the amount of information at each area (useful for next area) communicated to different vehicles, we focus on one coverage area (zone 1) and outside region (zone 0 ) only. Unlike $[15,19]$ in which the system model has multiple zones within the coverage area of an RSU with distinct transmission rates determined by the distances of the nodes from the RSU, our system model has only one zone within the coverage area of an RSU. We consider the highway to be multi lane, with $\mathrm{N}$ lanes, where lane $i$

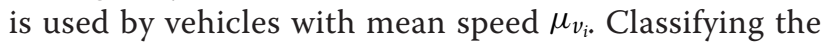
vehicles according to their mean speed, we have $\mathrm{N}$ classes of vehicles, a class $i$ vehicle has a mean speed $\mu_{v_{i}}$. Let $n_{i}$ be the no of vehicles belonging to class $i$. The probability density function of $V_{i}$, the random variable representing class $i$ vehicle velocity, is assumed to be uniform $[13,26]$ in the interval $\left(v_{\min , i}, v_{\max , i}\right)$, with $\mu_{v_{i}}$ representing the mean and $\sigma_{v_{i}}$ representing the standard deviation. Accordingly $v_{\max , i}=\mu_{v_{i}}+\sqrt{3} \sigma_{v_{i}}$ is the maximum speed and $v_{\min , i}=\mu_{v_{i}}-\sqrt{3} \sigma_{v_{i}}$ is the minimum speed. The pdf of $V_{i}$ is given by

$$
f_{V_{i}}\left(v_{i}\right)= \begin{cases}\frac{1}{2 \sqrt{3}} \frac{1}{\sigma_{v_{i}}} ; & \mu_{v_{i}}-\sqrt{3} \sigma_{v_{i}} \leq v_{i} \leq \mu_{v_{i}}+\sqrt{3} \sigma_{v_{i}} \\ 0 ; & \text { otherwise }\end{cases}
$$

The residence time of class $i$ vehicle in the coverage area of RSU is a random variable defined as $T_{1, i}=\frac{d_{1}}{V_{i}} ; i \in[1, N]$ where $d_{1}$ is the length of zone 1 . The mean sojourn time of class $i$ vehicle in the coverage area is calculated as follows:

$$
\begin{aligned}
E\left[T_{1, i}\right] & =d_{1} \frac{1}{E\left[V_{i}\right]}=d_{1} \int_{\mu_{v_{i}}-\sqrt{3} \sigma_{v_{i}}}^{\mu_{v_{i}}+\sqrt{3} \sigma_{v_{i}}} \frac{1}{v_{i}} \frac{1}{2 \sqrt{3} \sigma_{v_{i}}} d v_{i} \\
& =\frac{d_{1}}{2 \sqrt{3} \sigma_{v_{i}}} \ln \left(\frac{\mu_{v_{i}}+\sqrt{3} \sigma_{v_{i}}}{\mu_{v_{i}}-\sqrt{3} \sigma_{v_{i}}}\right)
\end{aligned}
$$

With this model, the highway could be of multiple unidirectional lanes and the vehicles are allowed to have variable speed, but move with constant mean velocity. A class $i$ vehicle entering zone1 resides in the coverage area of the RSU for a mean time duration $E\left[T_{1, i}\right]$ before moving out. The mobility of vehicles can then be represented by the zone transitions using a Markov chain model as shown in Figure 2. To facilitate the use of discrete time Markov chain model for the throughput analysis, the time that a class $i$ node stays in each zone $z$ is 


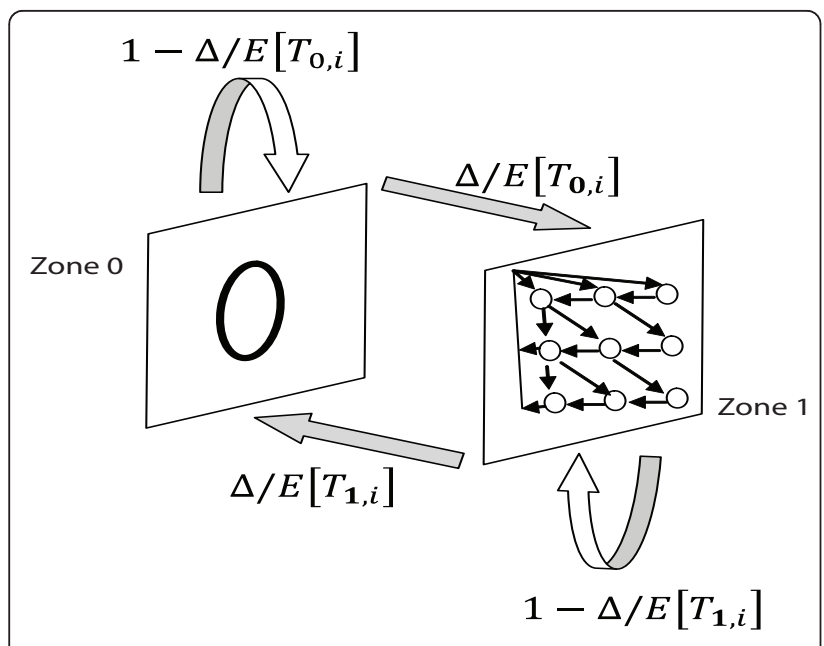

Figure 2 Markov chain model for zone transitions

approximated to be a geometrically distributed random variable with mean $E\left[T_{z, i}\right], z \in\{0,1\}$. Within a small duration, $\Delta$, vehicles belonging to class $i$ either move to zone 0 with probability $\frac{\Delta}{E\left[T_{1, i}\right]}$, or remain in zone 1 with probability $1-\frac{\Delta}{E\left[T_{1, i}\right]}$. The limiting probability that a node is in zone 1 at any time is given by $\frac{d_{1}}{d_{1}+d_{0}}$, where $d_{0}$ is the length of zone 0 .

When a vehicle is within the communication range of RSU, packet transmissions are coordinated by the DCF protocol. The packet length is assumed to be fixed and same for all nodes. Let $L$ ' be the maximum value of back off stage (assumed to be equal for all the nodes), such that for class $i$ node, the maximum value of $\mathrm{CW}$, $C W_{i, \max }=2^{L^{\prime}} W_{i, \mathrm{~min}}$. Let $W_{i, j}$ represent the CW in the $j$ th retry/retransmission for class $i$ node:

$$
W_{i, j}=\left\{\begin{array}{l}
2^{j} \times W_{i, \min } ; j=0,1, \ldots, L^{\prime}-1 \\
2^{L^{\prime}} \times W_{i, \min } ; j=L^{\prime}, \ldots, L
\end{array}\right.
$$

where $L$ denotes the maximum retry limit in DCF protocol.

\subsection{The discrete time Markov chain (DTMC) model for class $i$ vehicle}

To evaluate the throughput performance of DCF in V2I networks, we consider a tagged class $i$ vehicle and represents its states by a three-dimensional DTMC $\left\{z(t), s_{i}(t), b_{i}\right.$ $(t)\}$. Here $z(t)$ denotes the spatial zone in which the node is residing at time $t$. Clearly, $z(t)$ can take only one of the two values: 0 or $1 . s_{i}(t)$ and $b_{i}(t)$, respectively, denote the back-off stage and back-off counter of the tagged node at time $t$. Slot times $t$ and $t+1$ correspond to the beginning of two consecutive back-offs of the node. The duration of a time slot is a random variable as it may contain, an empty slot, a successful transmission slot or a collision slot
[7]. When the node is in zone 1 (within the coverage area of RSU), it follows DCF mechanism and the corresponding state transition diagram is shown in Figure 3.

Let $p_{c, i}$ and $\tau_{i}$, respectively, represent the frame collision probability (conditioned on a given class $i$ node transmission) and frame transmission probability for class $i$ node. As shown in Figure 3, when back off counter counts down to zero, the node would initiate the transmission attempt. If the transmission is collided, the node would back off by selecting a new back off time based on the DCF mechanism. In the case of a successful transmission the back off stage is cleared to zero. After each transmission attempt (failure or success), it is possible for the node to move to zone 0 or remain within zone 1 . Similarly after each decrement of back off counter, the tagged node either stays in the zone 1 or move to zone 0 . We consider that DCF is inactive in zone 0 (the back-off time set to infinity and the back-off stage cleared to 0 ), as in this case nodes are out of the transmission range. As such, nodes in zone 0 have only one state whereas those in zone 1 have multiple states with different values of back-off time and stage. The one-step transition probabilities ${ }^{\mathrm{a}}$ of the Markov chain from time slot $t$ to $t+1$ are as follows:

(i) State transition from zone 0 to zone 1:

$$
P(1,0, k \mid \mathcal{O})=\frac{E\left[T_{\text {slot }}\right]}{E\left[T_{0, i}\right] W_{i, \min }}, \quad k \in\left[0, W_{i, \min }-1\right](4)
$$

where $\mathcal{O}$ represents zone 0 and $E\left[T_{\text {slot }}\right]$ is the mean duration of one time slot. $P(1,0, k / \mathcal{O})$ accounts for the transition probability that the node moves from zone 0 to zone 1 and selects the back-off time $k$ from the range $\left[0, W_{i, \min }-1\right]$. This is because of the fact that within one time slot, with probability $\frac{E\left[T_{\text {slot }}\right]}{E\left[T_{0,1}\right]}$, the node moves from zone 0 to zone 1 according to the geometrically distributed sojourn time in each zone. After reaching zone 1 , the node selects the initial $b_{i}(t)$ uniformly from $\left[0, W_{i, \min }-1\right]$. As the zone transition and back-off time selection are independent, the overall transmission probability is $\frac{E\left[T_{\text {slot }}\right]}{E\left[T_{0,1}\right] W_{i, \min }}$.

(ii) State transitions within zone 1 (the RSU coverage):

Let $E\left[T_{s}\right]$ and $E\left[T_{c}\right]$, respectively, represent average successful and collision time of the class $i$ node in zone 1. The various transition probabilities are as follows:

$$
\begin{aligned}
P(1, j, k-1 \mid 1, j, k)= & \left(1-\frac{E\left[T_{\text {slot }}\right]}{E\left[T_{1, i}\right]}\right), \\
& j \in[0, L], k \in\left[1,2^{\min \left(j, L^{\prime}\right)} W_{i, \min }-1\right] \\
P(1, j, k \mid 1, j-1,0)= & \left(1-\frac{E\left[T_{c}\right]}{E\left[T_{1, i}\right]}\right)\left(\frac{p_{c, i}}{2^{j} W_{i, \min }}\right), \\
& j \in[1, L], k \in\left[0,2^{\min \left(j, L^{\prime}\right)} W_{i, \min }-1\right]
\end{aligned}
$$




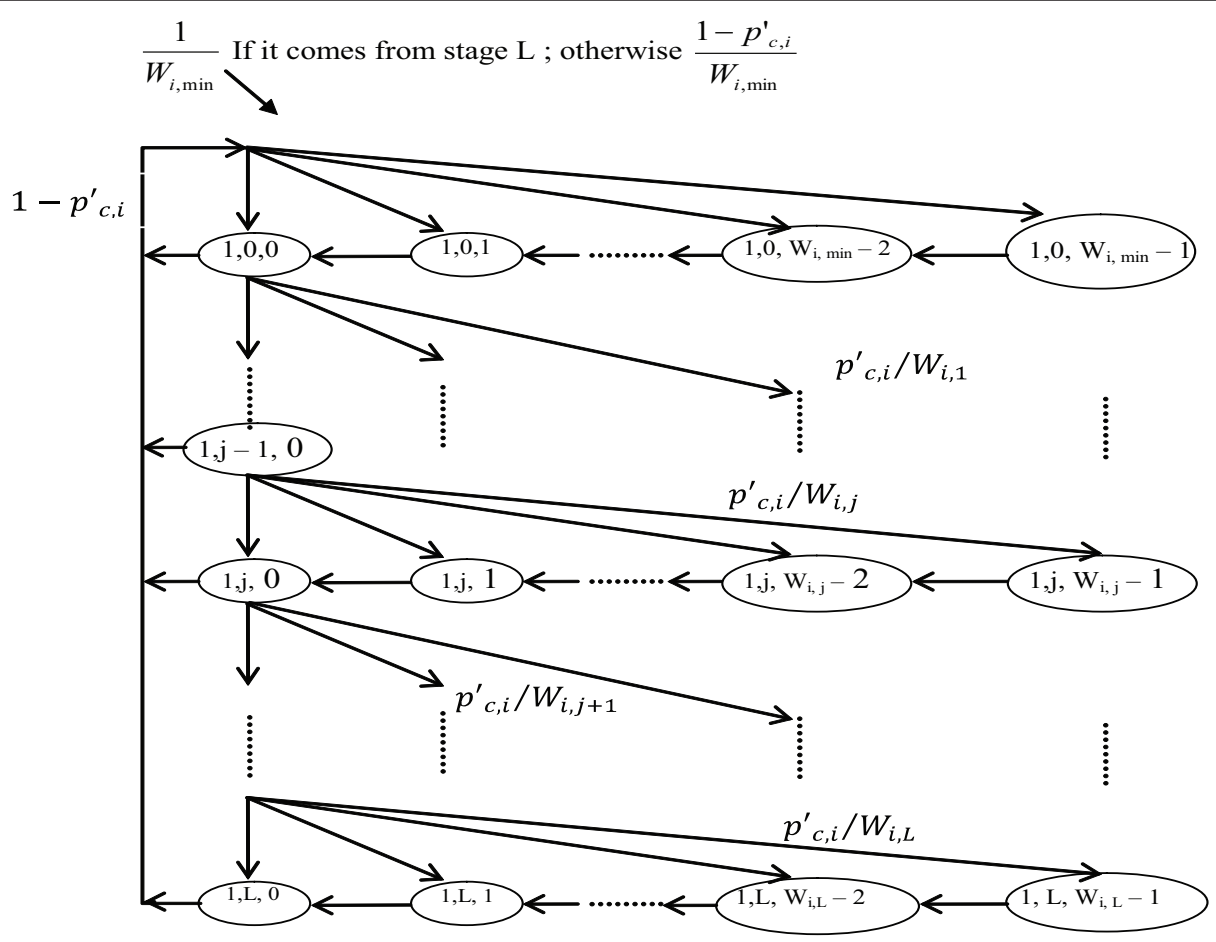

Figure 3 State transition diagram for class $i$ vehicle residing in the coverage area of RSU

$$
\begin{aligned}
& P(1,0, k \mid 1, j, 0)=\left(1-\frac{E\left[T_{s}\right]}{E\left[T_{1, i}\right]}\right) \frac{\left(1-p_{c, i}\right)}{W_{i, \min }}, \\
& j \in[0, L-1], k \in\left[0, W_{i, \min }-1\right] \\
& P(1,0, k \mid 1, L, 0)=\left(1-\left(\frac{p_{c, i} E\left[T_{c}\right]+\left(1-p_{c, i}\right) E\left[T_{s}\right]}{E\left[T_{1, i}\right]}\right)\right) \frac{1}{W_{i, \min }}, \quad(5 \mathrm{~d}) \\
& k \in\left[0,2^{L^{\prime}} W_{i, \mathrm{~min}}-1\right]
\end{aligned}
$$

Here (5a) accounts for the probability that the node remains in zone 1 after its back-off counter gets decremented by one. The second equation in (5b) accounts for the probability that the node encounters collision and enter the next back off stage, while remaining zone 1. The third equation (5c) accounts for the probability that the node transmits successfully and starts a new back-off and fourth case (5d) accounts for the probability that after the $L^{\text {th }}$ retransmission attempt, the node starts a new back off. Here $p_{c, i} E\left[T_{c}\right]+\left(1-p_{c, i}\right) E\left[T_{s}\right]$ represents the mean duration of transmission time (either successful or collided transmission).

(iii) State transition from zone 1 to zone 0 :

The transition probabilities that the node departs from zone 1 to zone 0 are given by

$$
P(\mathcal{O} \mid 1, j, k)=\frac{E\left[T_{\text {slot }}\right]}{E\left[T_{1, i}\right]}, \quad j \in[0, L], k \in\left[1,2^{\min \left(j, L^{\prime}\right)} W_{i, \min }-1\right]
$$

$$
P(\mathcal{O} \mid 1, j, 0)=\frac{\left(1-p_{c, i}\right) E\left[T_{s}\right]+p_{c, i} E\left[T_{c}\right]}{E\left[T_{1, i}\right]}, \quad j \in[0, L]
$$

Here (6) represents the probability that the tagged node departs zone 1 and enters zone 0 , after decrementing its back off counter, while (7) the probability that the transition from zone 1 to zone 0 occurs after a packet transmission attempt, where $\left(1-p_{c, i}\right) E\left[T_{s}\right]+p_{c, i} E$ $\left[T_{c}\right]$ is the mean duration of the transmission time. Since DCF protocol is inactive in zone 0 , the backoff counter value will not be inherited when the node enters the coverage area of the next RSU. Accordingly a fresh packet transmission will be initiated. It is assumed that the upper layer protocols will take care of the packets that are dropped during zone transitions. Define the stationary probability distribution of DTMC as follows

$$
\pi_{i}(z, j, k)=\lim _{t \rightarrow \infty} P\left\{z(t)=z, s_{i}(t)=j, b_{i}(t)=k\right\}
$$

where $z \in[0,1], j \in[0, L], k \in\left[0, W_{i, j}-1\right], i \in[1, N]$. The following relations can be obtained from the transition probabilities and the global balance equations:

$$
\begin{aligned}
\pi_{i}(1, j, 0) & =\left(1-\frac{E\left[T_{c}\right]}{E\left[T_{1, i}\right]}\right)^{j} p_{c, i}^{j} \pi_{i}(1,0,0) ; 0<j \leq L \\
& =\left(p_{c, i}^{\prime}\right)^{j} \pi_{i}(1,0,0) ; 0<j \leq L
\end{aligned}
$$




$$
\begin{aligned}
& \pi_{i}(1, j, k)=\left(\frac{W_{i, j}-k}{W_{i, j}}\right) \pi_{i}(1, j, 0) ; 0 \leq j \leq L, 1 \leq k \leq W_{i, j}-1 \\
& \sum_{j=0}^{L} \sum_{k=0}^{W_{i, j}-1} \pi_{i}(1, j, k)=\frac{d_{1}}{d_{1}+d_{0}}
\end{aligned}
$$

where $p_{c, i}^{\prime}=\left(1-\frac{E\left[T_{c}\right]}{E\left[T_{1, i}\right]}\right) p_{c, i}$ and (11) represents the limiting probability that a node resides within zone 1 . Combining (4) and (9)-(11), the following relation can be obtained for steady state probability $\pi_{i}(1,0,0)$ :

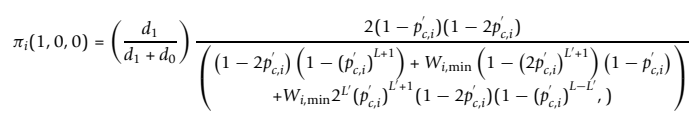

A frame transmission will occur when the back off counter is equal to zero, regardless of the back off stage, while the vehicle is in zone 1 . Here $\tau_{i}$ is the conditional probability that the class $i$ vehicle transmits a frame in a time slot, given that the vehicle is in zone 1:

$$
\begin{aligned}
\tau_{i} & =\frac{\sum_{j=0}^{L} \pi_{i}(1, j, 0)}{\left(\frac{d_{1}}{d_{1}+d_{0}}\right)}=\frac{\sum_{j=0}^{L}\left(p_{c, i}^{\prime}\right)^{j} \pi_{i}(1,0,0)}{\left(\frac{d_{1}}{d_{1}+d_{0}}\right)} \\
& =\left(\frac{1-\left(p_{c, i}^{\prime}\right)^{L+1}}{\left(1-p_{c, i}^{\prime}\right)}\right)\left(\frac{\pi_{i}(1,0,0)}{\left(\frac{d_{1}}{d_{1}+d_{0}}\right)}\right)
\end{aligned}
$$

Combining (12) and (13), we get the following expression for $\tau_{i}$ :

$$
\tau_{i}=\frac{2\left(1-\left(p_{c, i}^{\prime}\right)^{L+1}\right)\left(1-2 p_{c, i}^{\prime}\right)}{\left(\begin{array}{c}
\left(1-2 p_{c, i}^{\prime}\right)\left(1-\left(p_{c, i}^{\prime}\right)^{L+1}\right)+W_{i, \min }\left(1-\left(2 p_{c, i}^{\prime}\right)^{L^{\prime}+1}\right)\left(1-p_{c, i}^{\prime}\right) \\
+W_{i, \text { min }} 2^{L^{\prime}}\left(p_{c, i}^{\prime}\right)^{L^{\prime+1}}\left(1-2 p_{c, i}^{\prime}\right)\left(1-\left(p_{c, i}^{\prime}\right)^{L-L^{\prime}}\right)
\end{array}\right)}
$$

The conditional collision probability for the class $i$ node, $p_{c, i}$, can be expressed as,

$$
p_{c, i}=1-\left(1-\tau_{i}\right)^{n_{i}-1} \prod_{j=1, j \neq i}^{N}\left(1-\tau_{j}\right)^{n_{j}}
$$

Let $p_{t r}$ be the probability that at least one node transmits in a given slot time and is given by,

$$
p_{t r}=1-\prod_{j=1}^{N}\left(1-\tau_{j}\right)^{n_{j}}
$$

The probability $p_{s, i}$ that a class $i$ node transmits and it is successful is given by,

$$
p_{s, i}=\frac{n_{i} \tau_{i}\left(1-\tau_{i}\right)^{n_{i}-1} \prod_{j=1, j \neq i}^{N}\left(1-\tau_{j}\right)^{n_{j}}}{p_{t r}}
$$

The average successful payload information transmitted for class $i$ nodes that are within the coverage area of RSU is computed as follows

$$
\begin{aligned}
Z_{i} & =\frac{\left(\begin{array}{l}
\text { Average payload information for } \\
\text { class } i \text { transmitted in a slot time }
\end{array}\right)}{\text { Average length of a slot time }} \times \text { Mean residence time for class } i \\
Z_{i} & =\frac{p_{t r} p_{s, i} E[M]}{\left(1-p_{t r}\right) \sigma+p_{t r} p_{s} E\left[T_{s}\right]+p_{t r}\left(1-p_{s}\right) E\left[T_{c}\right]} \times E\left[T_{1, i}\right]
\end{aligned}
$$

where $E[M]$ is the average payload length (assumed to be equal for all nodes), $p_{s}$ is the probability that a transmission that occur in a time slot is successful, $\sigma$ is the duration of a empty time slot, $E\left[T_{1, i}\right]$ is the mean sojourn time for class $i$ within the coverage of RSU, $E$ $\left[T_{s}\right]$ and $E\left[T_{c}\right]$, respectively, represent the mean duration of successful and collision slots. Assuming basic access, these are computed as follows [7]:

$$
\begin{aligned}
& E\left[T_{s}\right]=T_{H}+T_{E[M]}+S I F S+\delta+T_{A C K}+D I F S+\delta \\
& E\left[T_{c}\right]=T_{H}+T_{E[M]}+D I F S+\delta
\end{aligned}
$$

Here $T_{H}, T_{E[M]}$ represent the transmission times of header and pay load, $\delta$ is the propagation delay, $T_{A C K}$ is the transmission time of the ACK packet and SIFS and DIFS are defined according to IEEE 802.11p standard. To compute the bits transferred for class $i$ node using (18), $\tau_{i}$ and $p_{c, i}$ are first determined using (14) and (15). It may be noted that (14) and (15) form a set of nonlinear equations which can be solved by using numerical techniques [7]. The bits transferred can be determined by using (16)-(18), if the no. of nodes corresponding to class $i, i \in(1, N)$ are given.

In V2I networks, the no. of vehicles on the highway depends on parameters such as vehicle arrival rate, vehicle density, and vehicle speed. The total arrival rate $\lambda_{i}$ of class $i$ vehicles to the RSU can be determined as

$$
\lambda_{i}=k_{i} \mu_{v_{i}}
$$

where $k_{i}$ is the vehicle density (veh/meter) in lane $i$ along the highway segment and $\mu_{v_{i}}$ is the mean vehicle speed (m/sec). According to Greenshield's model [23], the node density $k_{i}$ linearly changes with the mean velocity $\mu_{v_{i}}$ as

$$
k_{i}=k_{\text {jam }}\left(1-\frac{\mu_{v_{i}}}{v_{\text {free }}}\right)
$$

where $k_{\text {jam }}$ is the vehicle jam density at which traffic flow comes to a halt, $v_{\text {free }}$ is the free moving velocity, $i$. e., the maximum speed with which vehicle can move, when the vehicle is driving alone on the road (usually taken as the speed limit of the road). The mean number of class $i$ nodes, $N_{i}$ in the highway segment, is then determined using Little's theorem as follows [15]:

$$
\begin{aligned}
N_{i} & =\frac{\lambda_{i}\left(d_{1}+d_{0}\right)}{\mu_{v_{i}}} \\
& =k_{\text {jam }}\left(1-\frac{\mu_{v_{i}}}{v_{\text {free }}}\right)\left(d_{1}+d_{0}\right)
\end{aligned}
$$


The number of class $i$ nodes within the coverage area of RSU is given by

$$
\begin{aligned}
n_{i} & =N_{i} \frac{d_{1}}{d_{1}+d_{0}} \\
& =k_{\text {jam }}\left(1-\frac{\mu_{v_{i}}}{v_{\text {free }}}\right) d_{1}
\end{aligned}
$$

\section{Ensuring fairness in V2I networks}

As discussed earlier, the unfairness problem in V2I networks is caused by distinct velocities of the vehicles. A fast moving vehicle has less chance to communicate with its RSU, as compared to a slow moving vehicle, owing to their reduced residence time within RSU's coverage. Accordingly, the bits transferred of fast moving vehicle is less as compared to that of vehicles with lower speed. In this section, using the model developed in previous section, analytical expression for the optimal minimum CW required to achieve bit-based fairness is obtained. We use the following Jain's Fairness Index [27], in evaluating the fairness of channel access:

$$
F=\frac{\left(\sum_{i=1}^{U} y_{i}\right)^{2}}{U \sum_{i=1}^{U} y_{i}^{2}}
$$

where $U$ is the total number of nodes in the network, and $y_{i}$ 's are the individual node share. It may be noted that $F \leq 1$ and equality holds iff $y_{i}=y \forall i$.

\subsection{Selection of minimum $C W$ for fair service in V2I networks}

Our objective is to ensure that all competing nodes in the network achieve same amount of data transferred regardless of their velocities. Let $z_{i}=\frac{Z_{i}}{n_{i}}$ be the bits transferred per node for class $i$ and let $\sum_{i=1}^{N} n_{i}=U$ be the total number of nodes in the network. To ensure fairness, our aim is to achieve the following

$$
z_{j}=z, j=1,2,3, \ldots, N
$$

In the following section, we derive expression for the minimum $\mathrm{CW}$ required, for vehicles belonging to different classes of mean velocities, to meet the desired fairness objective. Initially we consider a network with two velocity classes. The analysis is then extended for a V2I network with three velocity classes.

\subsubsection{Two classes of mean velocities}

In the discussion that follows, the subscripts $S$ and $F$ correspond to classes of slow and fast vehicles, respectively. Let $n_{S}$ denote the number of slow moving vehicles and $n_{F}$ denote the number of fast moving vehicles. Also, let $\mu_{v_{S}}$ and $\mu_{v_{F}}$, respectively, denote the mean velocities of the slow and fast moving vehicles and let $E$ $\left[T_{1, S}\right]$ and $E\left[T_{1, F}\right]$, respectively, be mean values of their residence times. Further, let $W_{S \text {,min }}$ and $W_{F \text {,min }}$ be the minimum CW corresponding to two classes of velocities. Let the conditional frame transmission probabilities of slow and fast nodes be $\tau_{S}$ and $\tau_{F}$, respectively; and the corresponding collision probabilities $p_{c, S}$ and $p_{c, F}$. Using (14), $\tau_{S}$ and $\tau_{F}$ can be expressed in terms of these probabilities as:

$$
\begin{aligned}
& \tau_{S}=\frac{2\left(1-\left(p_{c, S}^{\prime}\right)^{L+1}\right)\left(1-2 p_{c, S}^{\prime}\right)}{\left(\begin{array}{c}
\left.\left(1-2 p_{c, S}^{\prime}\right)\left(1-\left(p_{c, S}^{\prime}\right)\right)^{L+1}\right)+W_{S, \min }\left(1-\left(2 p_{c, S}^{\prime}\right)^{L^{\prime}+1}\right)\left(1-p_{c, S}^{\prime}\right) \\
+W_{S, \text { min }} 2^{L^{\prime}}\left(p_{c, S}^{\prime}\right)^{L^{\prime}+1}\left(1-2 p_{c, S}^{\prime}\right)\left(1-\left(p_{c, S}^{\prime}\right)^{L-L^{\prime}}\right)
\end{array}\right)} \\
& \tau_{F}=\frac{2\left(1-\left(p_{c, F}^{\prime}\right)^{L+1}\right)\left(1-2 p_{c, F}^{\prime}\right)}{\left(\begin{array}{c}
\left(1-2 p_{c, F}^{\prime}\right)\left(1-\left(p_{c, F}^{\prime}\right)\right. \\
\left.+W_{F, \text { min }} 2^{L^{\prime}}\left(p_{c, F}^{\prime}\right){ }^{\prime}\right)+W_{F, \min }\left(1-\left(2 p_{c, F}^{\prime}\right)^{L^{\prime}+1}\right)\left(1-2 p_{c, F}^{\prime}\right)\left(1-\left(p_{c, F}^{\prime}\right)^{L-L^{\prime}}\right)
\end{array}\right)}
\end{aligned}
$$

where

$$
p_{c, S}^{\prime}=\left(1-\frac{E\left[T_{c}\right]}{E\left[T_{1, S}\right]}\right) p_{c, S}
$$

$p_{c, F}^{\prime}=\left(1-\frac{E\left[T_{c}\right]}{E\left[T_{1, F}\right]}\right) p_{c, F}$. Further, the collision probabilities $p_{c, S}$ and $p_{c, F}$ are expressed as

$$
\begin{aligned}
& p_{c, S}=1-\left(1-\tau_{S}\right)^{n_{S}-1}\left(1-\tau_{F}\right)^{n_{F}} \\
& p_{c, F}=1-\left(1-\tau_{F}\right)^{n_{F}-1}\left(1-\tau_{S}\right)^{n_{S}}
\end{aligned}
$$

Recall that $p_{t r}$ is the probability that there is at least one transmission in the given time slot, and let $p_{t r, S}$ and $p_{t r, F}$ be the corresponding probabilities for slow and fast nodes, respectively:

$$
\begin{aligned}
p_{t r} & =1-\left(1-\tau_{S}\right)^{n_{S}}\left(1-\tau_{F}\right)^{n_{F}} \\
p_{t r, S} & =1-\left(1-\tau_{S}\right)^{n_{S}} \\
p_{t r, F} & =1-\left(1-\tau_{F}\right)^{n_{F}}
\end{aligned}
$$

The success probabilities, as defined in (17), for two classes are:

$$
\begin{aligned}
p_{S, S} & =\frac{n_{S} \tau_{S}\left(1-\tau_{S}\right)^{n_{S}-1}\left(1-\tau_{F}\right)^{n_{F}}}{p_{t r}} \\
p_{S, F} & =\frac{n_{F} \tau_{F}\left(1-\tau_{F}\right)^{n_{F}-1}\left(1-\tau_{S}\right)^{n_{S}}}{p_{t r}}
\end{aligned}
$$

The amount of bits transferred, as defined in (18), for slow and fast moving vehicles are given by,

$$
\begin{aligned}
& Z_{S}=\frac{p_{t r} p_{s, S} E[M]}{\left(1-p_{t r}\right) \sigma+p_{t r} p_{s} E\left[T_{s}\right]+p_{t r}\left(1-p_{s}\right) E\left[T_{c}\right]} \times E\left[T_{1, S}\right] \\
& Z_{F}=\frac{p_{t r} p_{s, F} E[M]}{\left(1-p_{t r}\right) \sigma+p_{t r} p_{s} E\left[T_{s}\right]+p_{t r}\left(1-p_{s}\right) E\left[T_{c}\right]} \times E\left[T_{1, F}\right]
\end{aligned}
$$

Recall that the fairness index $\mathrm{F}$ becomes equal to unity when the bits transferred per node for slow and fast nodes are equal; i.e., $z_{S}=z_{F}$. From (28), we have $\left(1-p_{c, S}\right)\left(1-\tau_{S}\right)=\left(1-p_{c, F}\right)\left(1-\tau_{F}\right)=\left(1-\tau_{S}\right)^{n_{S}}\left(1-\tau_{F}\right)^{n_{F}}$. 
Assume $W_{S, \text { min }}, W_{F, \min }>>1$ and $\tau_{S}, \tau_{F}<<1$ so that $p_{c, S} \cong$ $p_{c, F}$. Then using (26), (27) and assuming the retry limit to be infinite, the following approximation is valid: $\frac{\tau_{S}}{\tau_{F}}=\frac{W_{F, \min }}{W_{S, \text { min }}}$. Utilizing (29), (30), and (31), we have the following approximation for ratio of bits transferred for slow and fast vehicles:

$$
\begin{aligned}
\frac{Z_{S}}{Z_{F}} & \cong \frac{n_{S} \tau_{S}\left(1-\tau_{S}\right)^{n_{S}-1}\left(1-\tau_{F}\right)^{n_{F}} E\left[T_{1, S}\right]}{n_{F} \tau_{F}\left(1-\tau_{F}\right)^{n_{F}-1}\left(1-\tau_{S}\right)^{n_{S}} E\left[T_{1, F}\right]} \\
& \cong \frac{n_{S} \frac{\tau_{S}}{1-\tau_{S}} E\left[T_{1, S}\right]}{n_{F} \frac{\tau_{F}}{1-\tau_{F}} E\left[T_{1, F}\right]} \cong \frac{n_{S} \tau_{S} E\left[T_{1, S}\right]}{n_{F} \tau_{F} E\left[T_{1, F}\right]}
\end{aligned}
$$

The ratio of the bits transferred per node is given by

$$
\frac{z_{S}}{z_{F}}=\frac{Z_{S} / n_{S}}{Z_{F} / n_{F}} \cong \frac{\tau_{S} E\left[T_{1, S}\right]}{\tau_{F} E\left[T_{1, F}\right]} \cong \frac{W_{F, \min } E\left[T_{1, S}\right]}{W_{S, \min } E\left[T_{1, F}\right]}
$$

Since $F=1$ when $z_{S}=z_{F}$, the optimal minimum CW for the fast vehicle to achieve desired fairness objective can be obtained as follows:

$$
W_{F, \min }^{*} \cong\left\lceil W_{S, \min } \frac{E\left[T_{1, F}\right]}{E\left[T_{1, S}\right]}\right\rceil
$$

Similarly, the optimal minimum CW for the slow vehicle can be computed, given the minimum $\mathrm{CW}$ of fast vehicle, as:

$$
W_{S, \min }^{*} \cong\left\lceil W_{F, \min } \frac{E\left[T_{1, S}\right]}{E\left[T_{1, F}\right]}\right\rceil
$$

Under the default parameter settings (where all MAC parameters are equal irrespective of node velocities), the ratio of bits transferred per node for slow and fast nodes is obtained from (33) as $z_{S} / z_{F} \cong E\left[T_{1, S}\right] / E\left[T_{1, F}\right]$. When optimal minimum $C W$ is chosen according to (34) or (35), the ratio of bits transferred per node for slow and fast station becomes equal to unity, thus resulting in bit-based fairness.

\subsubsection{Three classes of mean velocities}

In this section, we extend our analysis to a V2I network in which there are three classes of mean velocities: slow $(\mathrm{S})$, medium $(\mathrm{M})$ and fast $(\mathrm{F})$. Let $n_{S}, n_{M}, n_{F}$, respectively, denote the number of vehicles corresponding to the three categories. $\mu_{\nu_{S}}, \mu_{v_{M}}$, and $\mu_{v_{F}}$, respectively, be their mean velocities; and $E\left[T_{1, S}\right], E\left[T_{1, M}\right]$, and $E\left[T_{1, F}\right]$, respectively, be their mean residence time. Clearly, $E\left[T_{1}\right.$, $\left.{ }_{S}\right]>E\left[T_{1, M}\right]>E\left[T_{1, F}\right]$. Further, let $\tau_{S}, \tau_{M}$, and $\tau_{F}$ be the conditional frame transmission probabilities and let $p_{c, S}$, $p_{c, M}$, and $p_{c, F}$ be the frame collision probabilities of slow, medium and fast vehicles, respectively.

To ensure fairness, the minimum CW of medium and fast vehicles are decreased to improve their transmission opportunity during their residence within the coverage area of RSU. Keeping the minimum CW of slowest vehicle constant at default value, the optimal minimum $\mathrm{CW}$ pair $\left(W_{M, \min }^{*}, W_{F, \text { min }}^{*}\right)$ required to achieve $F=1$ is determined. Since the fairness index $F$ becomes equal to unity when $z_{S}=z_{M}=z_{F}$, where $z_{i}(i=S, M, F)$ represent the bits transferred per node for slow, medium and fast nodes respectively. Expressions similar to (33) can be obtained as

$$
\begin{aligned}
\frac{z_{S}}{z_{F}} \cong \frac{W_{F, \min } E\left[T_{1, S}\right]}{W_{S, \min } E\left[T_{1, F}\right]} \\
\frac{z_{S}}{z_{M}} \cong \frac{W_{M, \min } E\left[T_{1, S}\right]}{W_{S, \min } E\left[T_{1, M}\right]}
\end{aligned}
$$

Hence approximate expressions for optimal minimum CW pair for medium and fast nodes can be obtained as follows:

$$
\begin{aligned}
W_{M, \min }^{*} \cong\left\lceil W_{S, \min } \frac{E\left[T_{1, M}\right]}{E\left[T_{1, S}\right]}\right\rceil \\
W_{F, \min }^{*} \cong\left\lceil W_{S, \min } \frac{E\left[T_{1, F}\right]}{E\left[T_{1, S}\right]}\right\rceil
\end{aligned}
$$

Note that $W_{F, \text { min }}^{*}$ required to achieve bit-based fairness in a network with three classes of mean velocities is same as that of two classes case. Also, $W_{M \text {,min }}^{*}$ required to achieve bit based fairness in network with three classes of mean velocities is same as that required in a network two velocity classes, where the mean velocities are $\mu_{v_{M}}$ and $\mu_{v_{S}}$. Thus the optimal value of minimum $\mathrm{CW}$ required to achieve bit-based fairness in a network with two velocity classes, hold for network with three mean velocity classes as well. For a V2I network with N number of mean velocity classes, the results of (37) can be extended for all the higher velocity classes, provided we consider the slowest vehicle to be the reference node.

\section{Analytical and simulation results}

In this section, we present the analytical and simulation results. The analytical results correspond to the mathematical model presented in the previous section and are obtained using MATLAB. To validate the analytical results, we simulate a V2I network using an event driven custom simulation program, written in $\mathrm{C}++$ programming language, that closely follows the IEEE 802.11p standard: MAC layer based on EDCA and physical layer based on IEEE 802.11a. A drive-thru internet scenario, shown in the Figure 1, is simulated in which RSU is deployed along the road and vehicles passing through, compete for communication with RSUs. The whole road length is divided into two segments with one zone in the coverage area of RSU and other zone representing the region outside the coverage of RSU (we set $d_{1}=250 \mathrm{~m}$ and $\left.d_{0}=50 \mathrm{~m}\right)$. When there are two classes of vehicles, 
we simulate the road segment composed of two lanes. For the case of three classes, a three lane road segment is simulated. Vehicles arrive according to a poisson process with rate $\lambda$ veh/sec. Lane $i$ is used by vehicles belonging to class $i$ of mean velocity $v_{i}$. The probability distribution of $v_{i}$ is assumed to be uniform in the interval $\left(v_{\min , i}, v_{\max }\right.$, $\left.{ }_{i}\right)$ with $\mu_{v_{i}}$ representing the mean speed and $\sigma_{v_{i}}$, the standard deviation. We consider two values for traffic jam density $\left(k_{\mathrm{jam}}=80\right.$ and $\left.160 \mathrm{veh} / \mathrm{km} / \mathrm{lane}\right)$ and the free flow speed is selected as $v_{\text {free }}=160 \mathrm{~km} / \mathrm{hr}$ [28]. The data transmission rate is $6 \mathrm{Mbps}$. The system parameters used for simulation as well as for finding the numerical results are given in the in Table 1. All reported simulation results are averages over multiple $100 \mathrm{~s}$ simulations.

The number of vehicles corresponding to different classes of mean velocities, within the coverage area of RSU, are obtained using (23) with two values of $k_{\text {jam }}$ : 80 and $160 \mathrm{veh} / \mathrm{km} /$ lane. Table 2 lists the number of slow and fast vehicles in a network with two classes of mean velocities for different choices of mean velocities. Similar results are shown in Table 3 for a network with three classes of mean velocities. These results are later used to study the throughput performance of V2I networks.

\subsection{Network with two classes of mean velocities}

The MAC parameters for slow and fast vehicles are kept the same: $L=7, L^{\prime}=5, W_{S, \text { min }}=16$ or $32, W_{F, \text { min }}=16$ or 32. Further, we select $\mu_{v_{S}}=60 \mathrm{~km} / \mathrm{hr}, \mu_{v_{F}}=120 \mathrm{~km} / \mathrm{hr}$, $\sigma_{v_{F}}=5 \mathrm{~km} / \mathrm{hr}, \sigma_{v_{F}}=5 \mathrm{~km} / \mathrm{hr}, k_{\text {jam }}=80$ or $160 \mathrm{veh} / \mathrm{km} /$ lane and $v_{\text {free }}=160 \mathrm{~km} / \mathrm{hr}$. The number of vehicles corresponding to these specifications are listed in Table 2. We find the amount of data transferred (bits) for slow and fast vehicles by analysis using (31) as well as simulation. Here the analytical results are obtained using (31). The results are shown in Tables 4 and 5. We find that the data transferred for fast vehicles is very low compared to slow vehicles with default $C W_{\min }$ setting. Results are shown for two different values of $k_{\text {jam }}$ and two different values of mean velocities. The low data transfer for fast vehicle is caused by the DCF protocol which does not consider residence

Table 1 System parameters

\begin{tabular}{ll}
\hline Parameter & Value \\
\hline Packet payload & 8184 bits @6 Mb/s \\
MAC header & 256 bits @6 Mb/s \\
PHY header & 192 bits @ $3 \mathrm{Mb} / \mathrm{s}$ \\
ACK & 112 bits + PHY header @ 3 Mb/s \\
Channel bit rate & $6 \mathrm{Mb} / \mathrm{s}$ \\
Propagation delay & $2 \mu \mathrm{s}$ \\
Slot time & $13 \mu \mathrm{s}$ \\
SIFS & $32 \mu \mathrm{s}$ \\
DIFS & $58 \mu \mathrm{s}$ \\
\hline
\end{tabular}

Table 2 Network size: two classes of mean velocities

\begin{tabular}{|c|c|c|c|c|}
\hline \multirow[t]{2}{*}{$\overline{\mu_{v_{S}}} v_{F}(\mathrm{~km} / \mathrm{hr})$} & \multicolumn{2}{|c|}{${ }_{\mathrm{jam}}=80 \mathrm{veh} / \mathrm{km} /$ lane } & \multicolumn{2}{|c|}{$k_{\mathrm{jam}}=160 \mathrm{veh} / \mathrm{km} /$ lane } \\
\hline & $n_{s}$ & $n_{F}$ & $n_{s}$ & $n_{F}$ \\
\hline 60,120 & 12 & 5 & 25 & 10 \\
\hline 80,120 & 10 & 5 & 20 & 10 \\
\hline
\end{tabular}

time of a vehicle for granting channel access. Further, we observe that for default $C W_{\min }$ settings, the ratio of data transferred per node for slow and fast vehicles is equal to the ratio of their mean residence times. Our simulation results for the default $C W_{\text {min }}$ setting listed in Tables 4 and 5 corroborate the analytical results given in (33).

\subsubsection{Evaluation of optimal $\mathrm{CW}_{\min }$ for Slow and fast vehicles}

Continuing with the case, where there are two class of mean velocities, we try to achieve bit-based fairness by tuning $C W_{\min }$ of either slow or fast vehicles. Firstly, we keep $W_{F, \text { min }}=16, n_{S}=12, n_{F}=5$ (corresponding to $\mu_{v_{F}}=120 \mathrm{~km} / \mathrm{hr}, \mu_{v_{F}}=120 \mathrm{~km} / \mathrm{hr}, k_{\text {jam }}=80 \mathrm{veh} / \mathrm{km} /$ lane) and increase $W_{S \text {,min }}$, minimum CW of the slow vehicle. We evaluate the fairness index defined arlier for each $W_{S \text {, min }}$ and find that there is an optimal $W_{S, \text { min }}^{*}$ which makes Fairness index $F=1$, and its value is 30 (see Figure 4). The numerical solution of the equations from our analytical model also gives the optimal value to be equal to 30 . We change the numbers of the slow and fast vehicles and keep $n_{S}=25, n_{F}=10$ (corresponding to $\mu_{v_{S}}=60 \mathrm{~km} / \mathrm{hr}, \mu_{v_{F}}=120 \mathrm{~km} / \mathrm{hr}, k_{\text {jam }}=160$ veh/ km/lane) and find that the $W_{S \text {,min }}$ is the same and is independent of the numbers of the slow and fast vehicles in the network. The fairness index is plotted against the $W_{S \text {,min }}$ of the slow station in Figure 4. Optimal $W_{S, \text { min }}^{*}$ for different cases are plotted in Figure 5 . We find that the optimal values for the minimum $\mathrm{CW}$, to ensure fairness, strongly depend on the mean velocity of the vehicles. When $C W_{\min }$ of fast station is fixed, and velocity of slow vehicle is increased, the optimum minimum CW of slow station reduces. Consequently, to get fair access to system resources, its $C W_{\min }$ has to be decremented. It can also be seen from Figure 5 that, the optimal $C W_{\min }$ of slow vehicle is roughly inversely proportional to their mean speed; i.e., $W_{S, \min } \propto 1 / \mu_{v_{S}}$.

Next, we keep $W_{S \text {, min }}=16 ; n_{S}=12, n_{F}=5$, for $k_{\text {jam }}=$ 80 and vary $W_{F, \min }$, which is the $C W_{\min }$ of the fast vehicle. We evaluate the fairness index defined earlier

Table 3 Network size: three classes of mean velocities

\begin{tabular}{|c|c|c|c|c|c|c|}
\hline \multirow[t]{2}{*}{$\mu_{v_{S^{\prime}}} \mu_{v_{M^{\prime}}} v_{F}(\mathrm{~km} / \mathrm{hr})$} & \multicolumn{3}{|c|}{$\begin{array}{c}{ }_{\text {jam }}= \\
\text { lane }\end{array}$} & \multicolumn{3}{|c|}{$\begin{array}{c}k_{\text {jam }}=160 \mathrm{Veh} / \mathrm{km} / \\
\text { lane }\end{array}$} \\
\hline & $n_{S}$ & $n_{M}$ & $n_{F}$ & $n_{S}$ & $n_{M}$ & $n_{F}$ \\
\hline $40,80,120$ & 15 & 10 & 5 & 30 & 20 & 10 \\
\hline $30,90,150$ & 16 & 8 & 1 & 32 & 17 & 2 \\
\hline
\end{tabular}


Table 4 Data transferred (per node and total): default $C W_{\min }$ and optimal $\mathbf{C} W_{\text {min }}$ ( $\mu v_{S}=60 \mathrm{~km} / \mathrm{hr}, \mu_{v_{f}}=120 \mathrm{~km} / \mathrm{hr}, \sigma_{v_{s}}=\sigma_{v_{f}}=5 \mathrm{~km} / \mathrm{hr}, k_{\mathrm{jam}}=80$ and $160 \mathrm{veh} / \mathrm{km} / \mathrm{lane}$ )

\begin{tabular}{|c|c|c|c|c|c|c|c|c|}
\hline \multirow[t]{2}{*}{ Number of vehicles } & \multirow[t]{2}{*}{$C W_{\min }$ settings } & & \multicolumn{2}{|c|}{ Slow vehicle (Mb) } & \multicolumn{2}{|c|}{ Fast vehicle $(\mathrm{Mb})$} & \multicolumn{2}{|l|}{ Total (Mb) } \\
\hline & & & Analytical & Simulation & Analytical & Simulation & Analytical & Simulation \\
\hline & Default & $\begin{array}{l}W_{s, \text { min }}=16 \\
W_{f, \text { min }}=16\end{array}$ & 3.1035 & 3.0754 & 1.5517 & 1.5487 & 45.008 & 44.6495 \\
\hline$n_{S}=12$ & & $\begin{array}{l}W_{S, \text { min }}=32 \\
W_{f, \text { min }}=32\end{array}$ & 3.3499 & 3.3373 & 1.6749 & 1.6671 & 48.5738 & 48.3886 \\
\hline \multirow[t]{3}{*}{$n_{F}=5$} & Bit-based fairness & $\begin{array}{c}W_{S, \text { min }}^{*}=30 \\
W_{f, \text { min }}=16\end{array}$ & 2.5594 & 2.4681 & 2.5239 & 2.5765 & 42.7313 & 42.5433 \\
\hline & & $\begin{array}{c}W_{S, \text { min }}^{*}=62 \\
W_{f, \text { min }}=32\end{array}$ & 2.6636 & 2.6433 & 2.7026 & 2.7428 & 45.4772 & 45.5108 \\
\hline & Default & $\begin{array}{l}W_{S, \min }=16 \\
W_{F, \text { min }}=16\end{array}$ & 1.3442 & 1.3545 & 0.6710 & 0.6806 & 40.3263 & 40.6703 \\
\hline$n_{S}=12$ & & $\begin{array}{l}W_{s, \text { min }}=32 \\
W_{f, \text { min }}=32\end{array}$ & 1.4941 & 1.4863 & 0.7470 & 0.7317 & 44.8250 & 44.4757 \\
\hline \multirow[t]{3}{*}{$n_{F}=10$} & Bit-based fairness & $\begin{array}{c}W_{S, \text { min }}^{*}=30 \\
W_{f, \text { min }}=16\end{array}$ & 1.1130 & 1.0940 & 1.1267 & 1.1487 & 39.0941 & 38.8381 \\
\hline & & $\begin{array}{l}W_{S \min }=16 \\
W_{F, \text { min }}^{*}=9\end{array}$ & 1.3189 & 1.2732 & 1.3014 & 1.2602 & 45.9882 & 44.4360 \\
\hline & & $\begin{array}{c}W_{S, \text { min }}^{*}=62 \\
W_{F, \text { min }}=32\end{array}$ & 1.2259 & 1.2042 & 1.2286 & 1.2408 & 42.9354 & 42.5139 \\
\hline
\end{tabular}

for each $W_{F, \text { min }}$ and find that there is an optimal, $W_{F, \text { min }}^{*}$, which makes fairness index $F=1$, and its value is 9 (see Figure 6). The numerical solution of the equations from our analytical model also gives the same optimal value. We change the numbers of the slow and fast vehicles; i.e., $n_{S}=25, n_{F}=10$, for $k_{\text {jam }}=160$ and find that the optimal, $W_{F, \min }^{*}$, is the same, and hence we conclude that the $W_{F, \text { min }}^{*}$ is independent of the numbers of the slow and fast vehicles in the network. With the optimal values of $C W_{\min }$ of slow/fast vehicles, we evaluate the amount of data transferred for both slow and fast vehicles. The analytical results are obtained using
(31) and these are compared against the results obtained through simulation studies. Tables 4 and 5 list the results for various cases (the bit based fairness entries). Results of Table 4 are obtained for the following: $\mu_{v_{\mathrm{F}}}=120 \mathrm{~km} / \mathrm{hr}, \quad \mu_{v_{\mathrm{F}}}=120 \mathrm{~km} / \mathrm{hr}, \quad \sigma_{v_{S}}=5 \mathrm{~km} / \mathrm{hr}$, $\sigma_{v_{\mathrm{F}}}=5 \mathrm{~km} / \mathrm{hr}, k_{\mathrm{jam}}=80$ and $160 \mathrm{veh} / \mathrm{km} /$ lane, while those given in Table 5 are obtained for $\mu_{v_{S}}=80 \mathrm{~km} / \mathrm{hr}$, $\sigma_{v_{S}}=5 \mathrm{~km} / \mathrm{hr}, \sigma_{v_{S}}=5 \mathrm{~km} / \mathrm{hr}, \sigma_{v_{F}}=5 \mathrm{~km} / \mathrm{hr}, k_{\text {jam }}=80$ and $160 \mathrm{veh} / \mathrm{km} / \mathrm{lane}$.

It can be observed that, with optimal $C W_{\min }$ values, the data transferred for the nodes are almost equal irrespective of their velocities; thus ensuring bit-based

Table 5 Data transferred (per node and total): default $\mathbf{C W}_{\min }$ and optimal $\mathrm{CW}_{\min }$

$\left(\mu_{\mathrm{v}_{\mathrm{S}}}=80 \mathrm{~km} / \mathrm{hr}, \mu \mathrm{v}_{\mathrm{F}}=120 \mathrm{~km} / \mathrm{hr}, \sigma_{\mathrm{v}_{\mathrm{S}}}=\sigma_{\mathrm{v}_{\mathrm{F}}}=5 \mathrm{~km} / \mathrm{hr}, \mathrm{k}_{\mathrm{jam}}=80\right.$ and $160 \mathrm{veh} / \mathrm{km} / \mathrm{lane}$ )

\begin{tabular}{|c|c|c|c|c|c|c|c|c|}
\hline \multirow[t]{2}{*}{ Number of vehicles } & \multicolumn{2}{|c|}{$C W_{\min }$ Settings } & \multicolumn{2}{|c|}{ Slow vehicle $(\mathrm{Mb})$} & \multicolumn{2}{|c|}{ Fast vehicle $(\mathrm{Mb})$} & \multicolumn{2}{|c|}{ Total (Mb) } \\
\hline & & & Analytical & Simulation & Analytical & Simulation & Analytical & Simulation \\
\hline & Default & $\begin{array}{l}W_{S, \text { min }}=16 \\
W_{F, \text { min }}=16\end{array}$ & 2.6806 & 2.6829 & 1.7870 & 1.7749 & 35.7415 & 35.7043 \\
\hline$n_{S}=10$ & & $\begin{array}{l}W_{S, \min }=32 \\
W_{F, \min }=32\end{array}$ & 2.8965 & 2.8893 & 1.9376 & 1.8891 & 38.7538 & 38.3355 \\
\hline \multirow[t]{3}{*}{$n_{F}=5$} & Bit-based fairness & $\begin{array}{c}W_{S, \text { min }}^{*}=23 \\
W_{F, \text { min }}=16\end{array}$ & 2.3618 & 2.3313 & 2.3679 & 2.3837 & 35.4588 & 35.2317 \\
\hline & & $\begin{array}{c}W_{S, \text { min }}^{*}=47 \\
W_{F, \text { min }}=32\end{array}$ & 2.5426 & 2.5071 & 2.5662 & 2.5551 & 38.2578 & 37.8477 \\
\hline & Default & $\begin{array}{l}W_{S, \text { min }}=16 \\
W_{F, \text { min }}=16\end{array}$ & 1.2076 & 1.2223 & 0.8050 & 0.8032 & 32.2028 & 32.4785 \\
\hline$n_{S}=20$ & & $\begin{array}{l}W_{S, \text { min }}=32 \\
W_{F, \text { min }}=32\end{array}$ & 1.3351 & 1.3359 & 0.8900 & 0.8803 & 35.6032 & 35.2223 \\
\hline \multirow[t]{2}{*}{$n_{F}=5$} & Bit-based fairness & $\begin{array}{c}W_{S, \text { min }}^{*}=23 \\
W_{F, \text { min }}=16\end{array}$ & 1.0797 & 1.0771 & 1.0630 & 1.0663 & 32.2245 & 32.2068 \\
\hline & & $\begin{array}{c}W_{S, \text { min }}^{*}=47 \\
W_{F, \text { min }}=32\end{array}$ & 1.1787 & 1.1609 & 1.1800 & 1.1920 & 35.3755 & 35.1402 \\
\hline
\end{tabular}




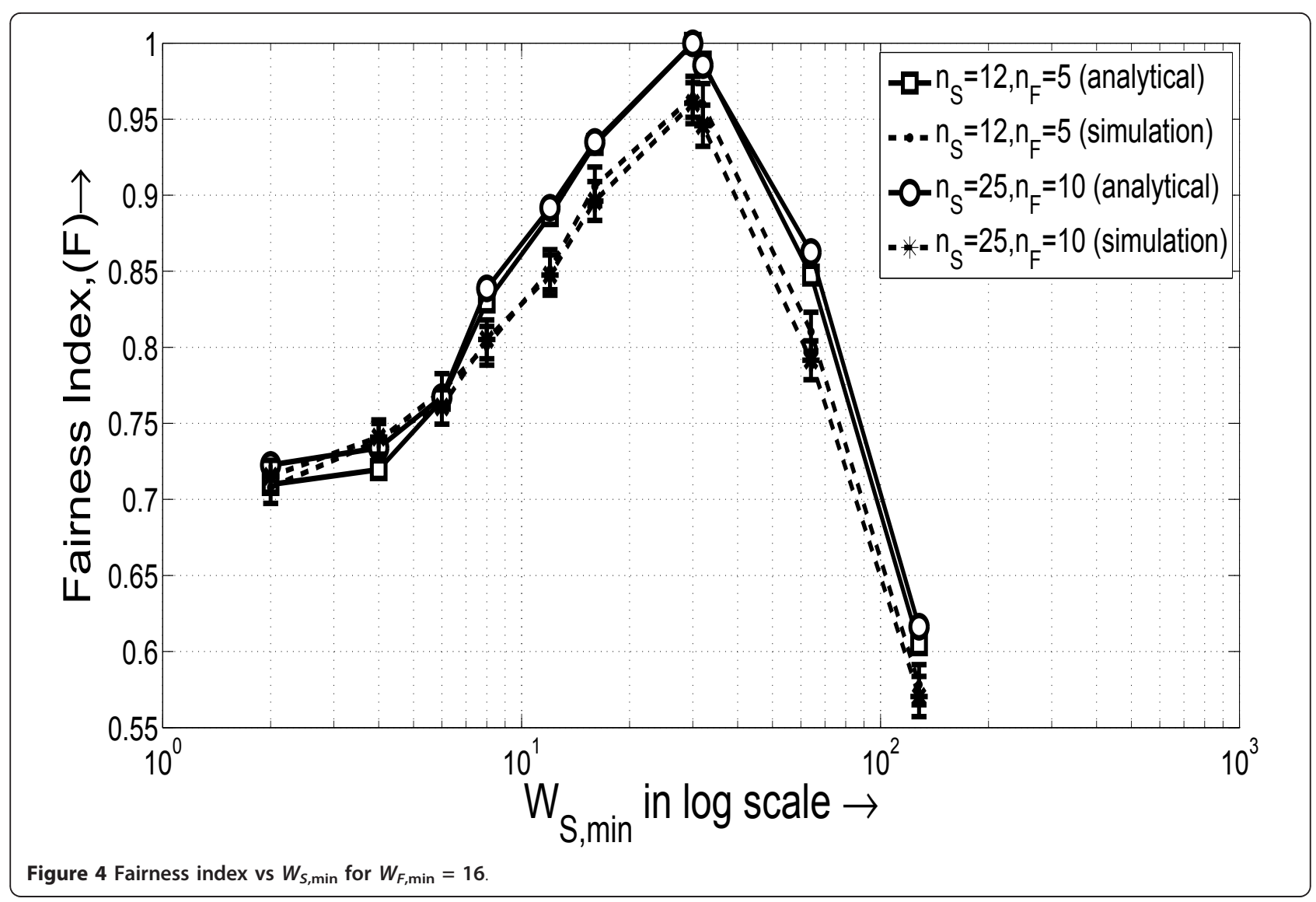

fairness. Further, the optimal $C W_{\min }$ values do not depend on the number of slow or fast stations, thus bitbased fairness is maintained always, irrespective of the network size. However, we observe a slight reduction in the total amount of data transferred (last column in Tables 4 and 5) for the bit-based fairness case compared to the default case. One possible reason for this reduction is that we used default value for $W_{F \text {,min }}$ and optimal value for $W_{S \text {,min }}$ which is larger than the corresponding default. On the other hand, when we used default value for $W_{S \text {,min }}$ and optimal value for $W_{F \text {,min }}$ which is smaller than the default, we got improved value. In our future study, we will be choosing optimal values for the windows of all classes, so as to maximize the total data transferred while also providing the bit-based fairness.

Fgure 7 shows the ratio of data transferred per node for fast and slow vehicle $\left(z_{\mathrm{F}} / z_{S}\right)$ plotted against the mean velocity of slow vehicle $\left(\mu_{v_{S}} \mathrm{~km} / \mathrm{hr}\right)$. Under the default settings, we select $W_{S, \text { min }}=W_{F, \text { min }}=16$, $\sigma_{v_{S}}=\sigma_{v_{F}}=5 \mathrm{~km} / \mathrm{hr}, \sigma_{\nu_{S}}=\sigma_{v_{F}}=5 \mathrm{~km} / \mathrm{hr}$, and vary $\mu_{v_{S}}$ from 20 to $120 \mathrm{~km} / \mathrm{hr}$. In this case, the ratio $z_{F} / z_{S}=$ increases as $\mu_{v_{S}}$ increases. This happens because, as $\mu_{v_{S}}$ increases, its residence time within RSU's coverage decreases and hence $z_{S}$ decreases. If the $C W_{\min }$ of slow vehicle is adapted to its mean velocity by selecting $W_{S, \text { min }}^{*}$ according to (35), both the slow and fast vehicles are assured to get same chances of communication with the RSU.

\subsection{Network with three classes of mean velocities}

We set mean velocities as: $\mu_{v_{S}}=40 \mathrm{~km} / \mathrm{hr}$, $\mu_{v_{F}}=120 \mathrm{~km} / \mathrm{hr}, \quad \mu_{v_{F}}=120 \mathrm{~km} / \mathrm{hr}$; $\sigma_{v_{S}}=\sigma_{v_{M}}=\sigma_{v_{F}}=5 \mathrm{~km} / \mathrm{hr}, k_{\text {jam }}=80 \mathrm{veh} / \mathrm{km} /$ lane and $v_{\text {free }}=160 \mathrm{~km} / \mathrm{hr}$. The number of slow $/$ medium $/$ fast vehicles corresponding to these traffic parameters are listed in Table 3. To find the optimal $C W_{\min }$ for slow and medium velocity vehicles, we keep $W_{F \text {,min }}$ fixed (16 or 32) and increase $W_{S \text {,min }}$ and $W_{M \text {,min }}$. The optimal pair $\left(W_{S, \text { min }}^{*} W_{M, \min }^{*}\right)$ which makes $F=1$, is obtained as $(46,24)$ for $W_{F, \text { min }}=16$ and as $(92,47)$ for $W_{F, \text { min }}=32$. The results are shown in Table 6 which also show that the optimal $C W_{\min }$ pair that achieve $F=1$ is independent of number of vehicles in the network. With these optimal $C W_{\min }$ values, we evaluate the data transferred for slow, medium and fast vehicles; the same also for the default setting of $C W_{\min }$ values. The analytical results corresponding to these are determined by using (31). Both the analytical and simulation results are 


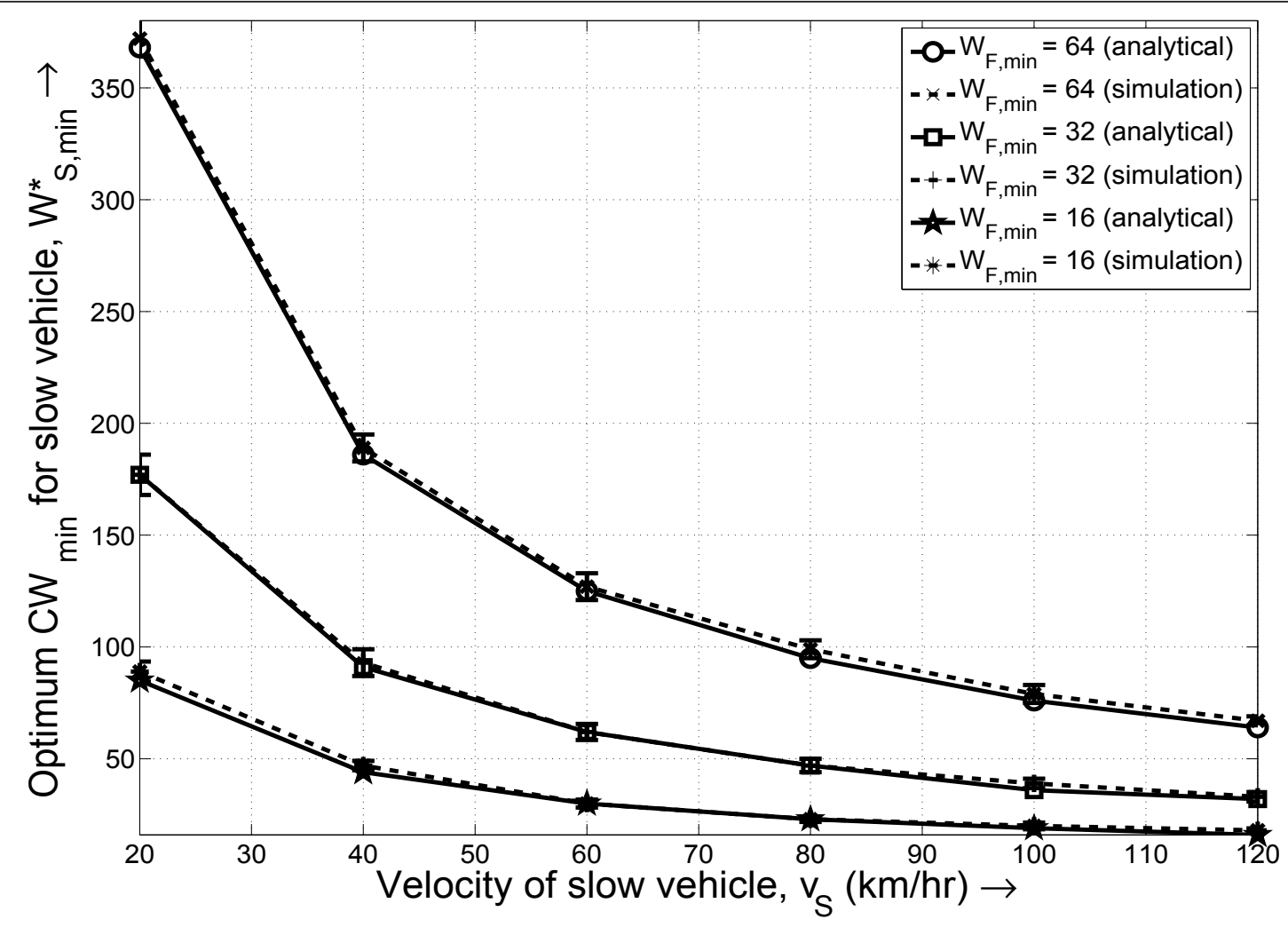

Figure 5 Variation of optimal $C W_{\min }$ of slow vehicle $\left(W_{S, \min }^{*}\right)$ with $\mu_{v_{S}}$ for $\mu_{v_{F}}=120 \mathrm{~km} / \mathrm{hr}$ and a given $W_{F, \text { min }}$

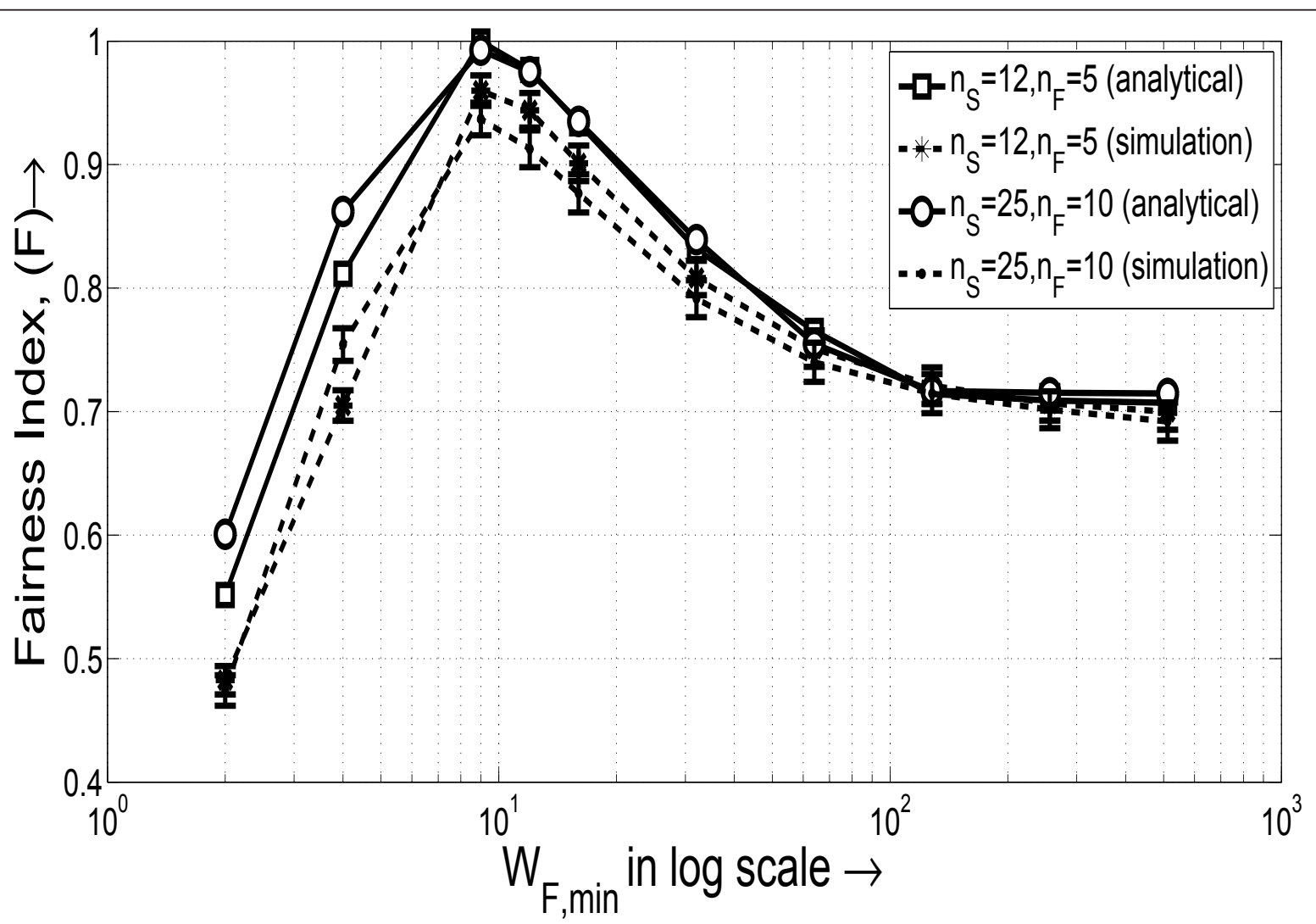




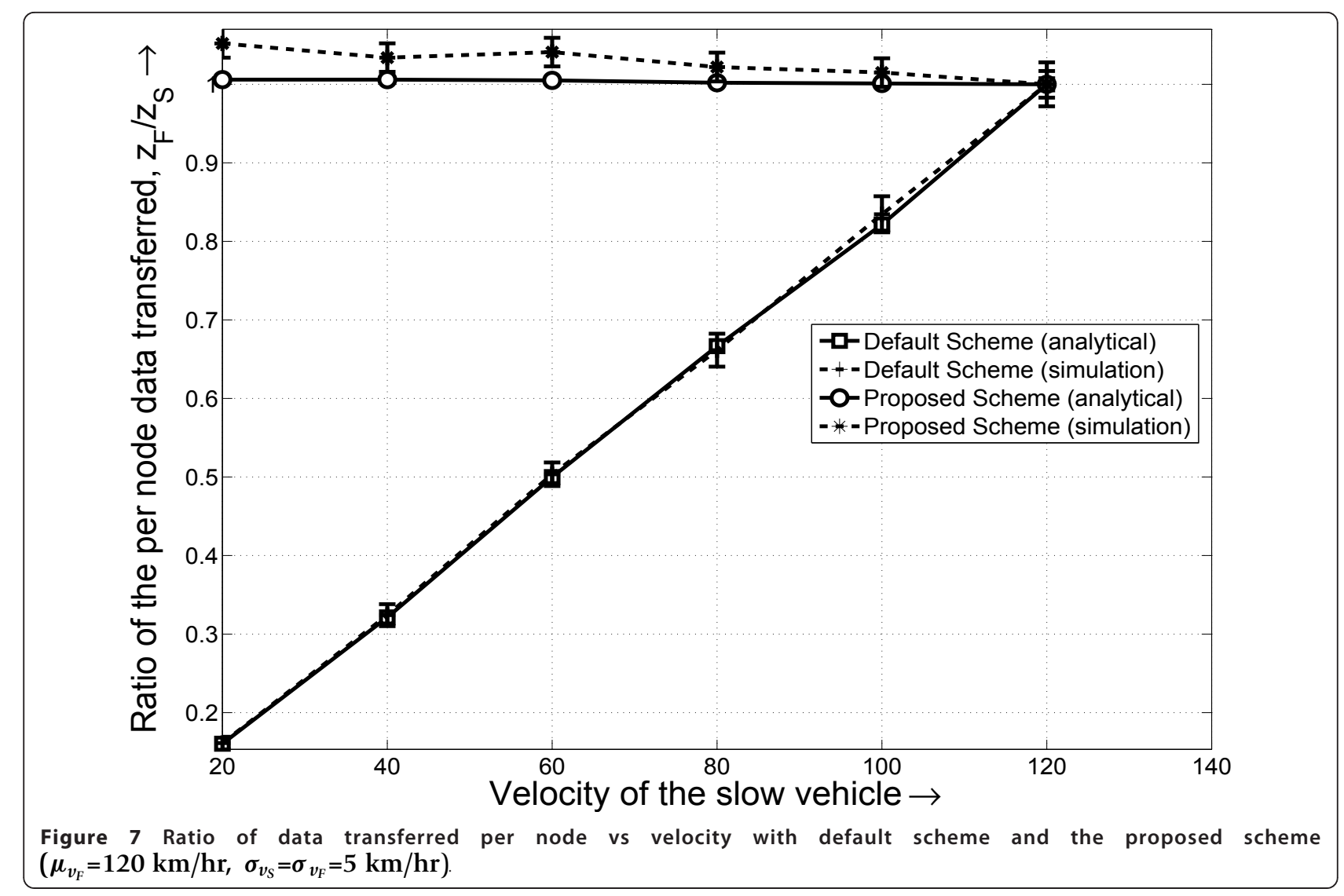

shown in Table 7. The evaluation is repeated for another set of mean velocities: $\mu_{v_{s}}=80 \mathrm{~km} / \mathrm{hr}$, $\mu_{v_{\mathrm{F}}}=140 \mathrm{~km} / \mathrm{hr}$,

(with $\sigma_{v_{S}}=\sigma_{v_{M}}=\sigma_{v_{F}}=5 \mathrm{~km} / \mathrm{hr}$ )-

(with $\sigma_{v_{S}}=\sigma_{v_{M}}=\sigma_{v_{F}}=5 \mathrm{~km} / \mathrm{hr}$ ); and the results are tabulated in Table 7. For the default selection of $C W_{\min }$, we find that the data transferred for fast and medium velocity vehicles are very less compared to that of the slow vehicle. Further, the ratio is equal to the ratio of corresponding mean residence times, which validates

Table 6 Variation of fairness index with $C W_{\min }$ of slow and medium Vehicle for $W_{F, \min }=16$

\begin{tabular}{|c|c|c|c|c|c|}
\hline \multicolumn{2}{|c|}{ Window size } & \multicolumn{4}{|c|}{ Fairness index } \\
\hline \multirow[t]{2}{*}{$W_{M, \min }$} & \multirow[t]{2}{*}{$W_{S, \min }$} & \multicolumn{2}{|c|}{$n_{F}=5, n_{M}=10, n_{S}=15$} & \multicolumn{2}{|c|}{$n_{F}=10, n_{M}=20, n_{S}=30$} \\
\hline & & Analysis & Simulation & Analysis & Simulation \\
\hline 4 & 4 & 0.7960 & 0.7633 & 0.7949 & 0.7549 \\
\hline 8 & 8 & 0.8223 & 0.7846 & 0.8217 & 0.7745 \\
\hline 16 & 16 & 0.8681 & 0.8314 & 0.8677 & 0.8205 \\
\hline 24 & 24 & 0.9017 & 0.8703 & 0.9013 & 0.8671 \\
\hline 24 & 46 & 0.9998 & 0.9618 & 0.9998 & 0.9502 \\
\hline 32 & 32 & 0.9213 & 0.8732 & 0.9211 & 0.8627 \\
\hline 64 & 64 & 0.8822 & 0.8318 & 0.8862 & 0.8162 \\
\hline 128 & 128 & 0.6504 & 0.5986 & 0.6504 & 0.5915 \\
\hline
\end{tabular}

our approximation given by (36). With optimal $C W_{\min }$ values, all the vehicles in the network achieve almost equal share, irrespective of their mean velocities; thus ensuring bit-based fairness.

\subsection{Impact of standard deviation of vehicle speed}

In Figure 8, we plot optimal $C W_{\min }$ against standard deviation of vehicle speed. We consider a network with two classes of mean velocities: $\mu_{v_{S}}=80 \mathrm{~km} / \mathrm{hr}$, $\mu_{v_{M}}=120 \mathrm{~km} / \mathrm{hr}$. We fix the standard deviation of velocity of fast vehicle $\sigma_{v_{F}}=5 \mathrm{~km} / \mathrm{hr}$ and vary $\sigma_{v_{S}}$, the standard deviation of slow vehicle velocity. We determine the impact of vehicle speed variability on optimal $C W_{\text {min }}$ of slow vehicle. When the standard deviation of vehicle speed increases, the mean residence time of slow velocity vehicle, $E\left[T_{1, S}\right]$ increases slightly. Accordingly the optimal $C W_{\min }$ also increases slightly. It is observed that the increase of $C W_{\min }$ is insignificant when $\sigma_{v_{S}}$ varies from 5 to $35 \mathrm{~km} / \mathrm{hr}$.

Figure 9 shows the impact of standard deviation of vehicle speed corresponding to slow moving vehicle on the ratio of data transferred per node for fast and slow vehicle $\left(z_{F} / z_{S}\right)$. For the default setting, we select $W_{S \text {, min }}=$ $W_{F, \text { min }}=16$. In this case, the ratio of data transferred is approximately equal to the ratio of their mean residence 
Table 7 Data transferred (per node and total): default $C W_{\min }$ and optimal

$C W_{\min }\left(\mu_{v_{s}}=40 \mathrm{~km} / \mathrm{hr}, \mu_{v_{M}}=80 \mathrm{~km} / \mathrm{hr}, \mu_{v_{F}}=120 \mathrm{~km} / \mathrm{hr}, k_{\mathrm{jam}}=80 \mathrm{veh} / \mathrm{km} / \mathrm{lane}\right.$ and $\left.\mu_{v_{s}}=80 \mathrm{~km} / \mathrm{hr}, \mu_{v_{M}}=105 \mathrm{~km} / \mathrm{hr}, \mu_{v_{f}}=140 \mathrm{~km} / \mathrm{hr}, k_{\mathrm{jam}}=80 \mathrm{veh} / \mathrm{km} / \mathrm{lane}, \sigma_{v_{\mathrm{s}}}=\sigma_{v_{M}}=\sigma_{v_{F}}=5 \mathrm{~km} / \mathrm{hr},\right)$

\begin{tabular}{|c|c|c|c|c|c|c|c|c|c|c|}
\hline \multirow{2}{*}{$\begin{array}{l}\text { Number of } \\
\text { vehicles }\end{array}$} & & \multirow{2}{*}{$\begin{array}{l}C W_{\min } \\
\text { Settings }\end{array}$} & \multicolumn{2}{|c|}{ Slow vehicle $(\mathrm{Mb})$} & \multicolumn{2}{|c|}{ Medium vehicle (Mb) } & \multicolumn{2}{|c|}{ Fast vehicle (Mb) } & \multicolumn{2}{|c|}{ Total (Mb) } \\
\hline & & & Analytical & Simulation & Analytical & Simulation & Analytical & Simulation & Analytical & Simulation \\
\hline$n_{S}=15$ & Default & $\begin{array}{l}W_{S, \text { min }}=16 \\
W_{M, \text { min }}=16 \\
W_{f, \text { min }}=16\end{array}$ & 2.4152 & 2.3398 & 1.2070 & 1.1592 & 0.8050 & 0.7728 & 52.3294 & 50.5451 \\
\hline$n_{M}=10$ & & $\begin{array}{l}W_{S, \text { min }}=32 \\
W_{M, \text { min }}=32 \\
W_{F, \text { min }}=32\end{array}$ & 2.6702 & 2.5213 & 1.3351 & 1.2751 & 0.8900 & 0.8330 & 57.8550 & 54.7367 \\
\hline \multirow[t]{2}{*}{$n_{F}=5$} & $\begin{array}{l}\text { Bit-based } \\
\text { fairness }\end{array}$ & $\begin{array}{c}W_{S, \text { min }}^{*}=46 \\
W_{M, \text { min }}^{*}=24 \\
W_{f, \text { min }}=16\end{array}$ & 1.5682 & 1.4642 & 1.5565 & 1.4903 & 1.6187 & 1.5509 & 47.1824 & 44.6424 \\
\hline & & $\begin{array}{c}W_{S, \text { min }}^{*}=92 \\
W_{M, \text { min }}^{*}=47 \\
W_{f, \text { min }}=32\end{array}$ & 1.7066 & 1.5730 & 1.7151 & 1.6187 & 1.7243 & 1.6521 & 51.3728 & 48.8124 \\
\hline$n_{S}=10$ & Default & $\begin{array}{l}W_{S, \text { min }}=16 \\
W_{M, \text { min }}=16 \\
W_{f, \text { min }}=16\end{array}$ & 2.1775 & 2.0989 & 1.6590 & 1.5998 & 1.2444 & 1.1899 & 34.2181 & 32.9676 \\
\hline$n_{M}=6$ & & $\begin{array}{l}W_{S, \text { min }}=32 \\
W_{M, \text { min }}=32 \\
W_{f, \text { min }}=32\end{array}$ & 2.3719 & 2.3211 & 1.8071 & 1.7888 & 1.3553 & 1.3212 & 37.2734 & 36.5862 \\
\hline \multirow[t]{2}{*}{$n_{F}=2$} & $\begin{array}{c}\text { Biot-based } \\
\text { fairness }\end{array}$ & $\begin{array}{c}W_{S, \text { min }}^{*}=28 \\
W_{M, \text { min }}^{*}=22 \\
W_{f, \text { min }}=16\end{array}$ & 1.8168 & 1.7918 & 1.8001 & 1.7788 & 1.9010 & 1.7912 & 32.9506 & 32.1732 \\
\hline & & $\begin{array}{l}W_{S, \min }^{*}=56 \\
W_{M, \text { min }}^{*}=44 \\
W_{f, \text { min }}=32\end{array}$ & 1.9813 & 1.9711 & 1.9474 & 1.9299 & 1.9166 & 1.9098 & 35.3306 & 35.0123 \\
\hline
\end{tabular}

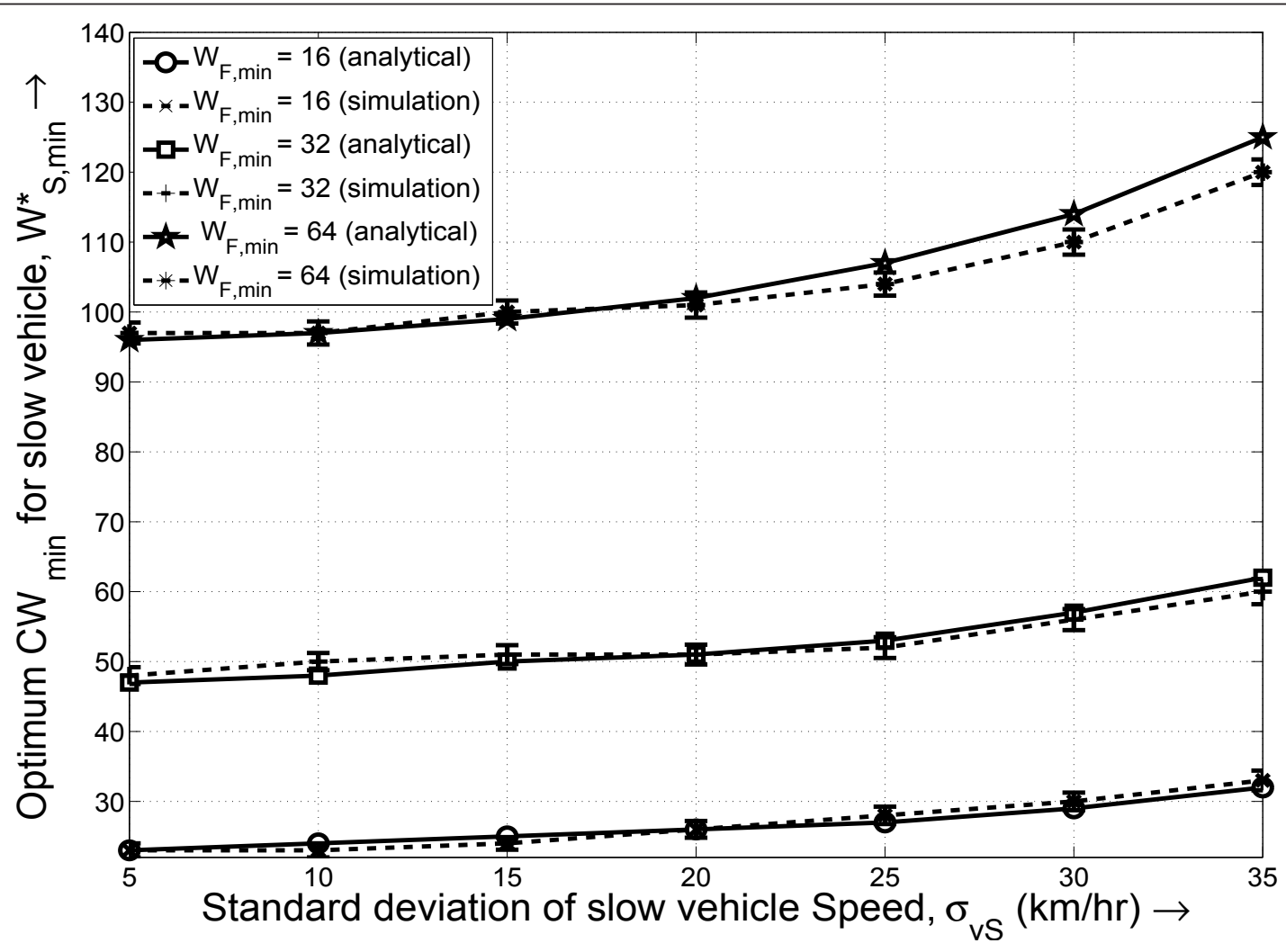

Figure 8 Optimal $C W_{\min }$ for slow vehicle vs standard deviation of slow vehicle speed $\left(\mu_{\mathrm{vs}_{\mathrm{S}}}=80 \mathrm{~km} / \mathrm{hr}, \mu_{\mathrm{vF}_{\mathrm{F}}}=120 \mathrm{~km} / \mathrm{hr}, \sigma_{\mathrm{v}_{\mathrm{F}}}=5 \mathrm{~km} / \mathrm{hr}\right)$. 


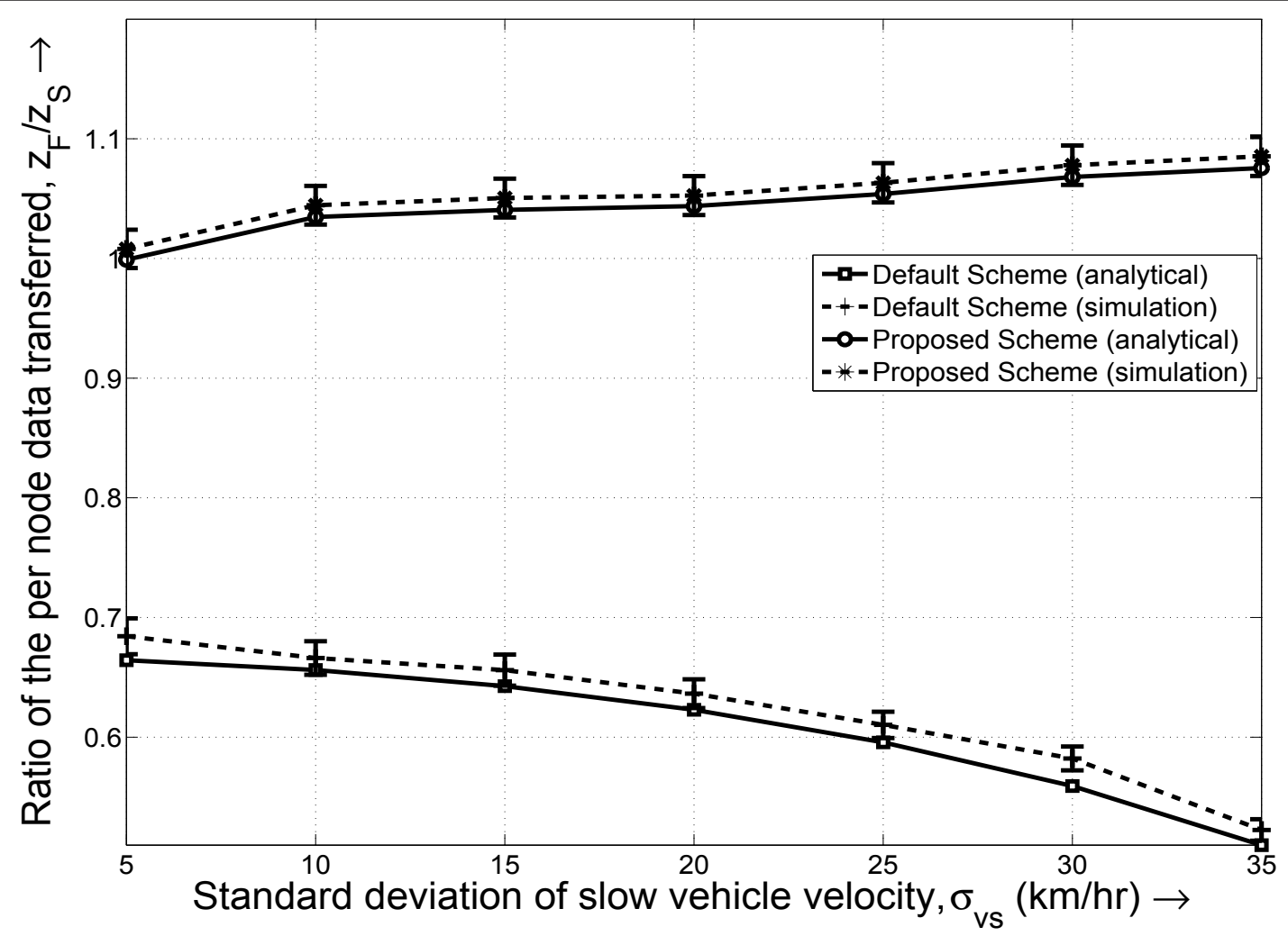

Figure 9 Ratio of data transferred per node vs standard deviation of slow vehicle speed with default scheme and the proposed scheme $\left(\mu_{\mathrm{v}_{\mathrm{S}}}=80 \mathrm{~km} / \mathrm{hr}, \mu_{\mathrm{v}_{\mathrm{F}}}=120 \mathrm{~km} / \mathrm{hr}, \sigma_{\mathrm{v}_{\mathrm{F}}}=5 \mathrm{~km} / \mathrm{hr}\right)$.

times. As the standard deviation increases, $E\left[T_{1, S}\right]$ increases, so $z_{F} / z_{S}$ decreases, as $z_{s}$ increases. With optimal $C W_{\min }$ settings, both slow and fast vehicles are assured to get equal chances of communication with the RSU and hence the ratio $z_{F} / z_{s}$ has to be unity. However, when $\sigma_{v_{S}}$ increases, the ratio $z_{F} / z_{S}$ slightly deviates from unity; owing to the moderate change in optimal $C W_{\min }$ arising out of variability of slow velocity vehicle.

\section{Conclusion}

In this article, we have investigated the issue of fairness in IEEE 802.11 -based vehicle to Infrastructure networks. We presented a simple yet accurate analytical model to compute the data transferred for contending vehicles in V2I network, taking into account their residence time within the coverage area of RSU. Classifying the vehicles according to their mean velocities, the model can be used to find the data transferred for a class $i$ vehicle with mean velocity $\mu_{v_{i}}, i \in[1, N]$. We, then addressed an unfairness problem that occur in V2I networks because of distinct vehicle velocities. The communication with RSU for a vehicle with higher velocity is affected significantly owing to their reduced residence time within the coverage area of RSU. It was proved that ratio of data transferred for vehicles with two different mean velocities is equal to the ratio of their mean residence times, assuming that the vehicles use the same MAC parameters and frame size. This implies that average amount of data transferred for each node is inversely proportional to its velocity, meaning that a fast moving vehicle, which naturally needs more recent information, has less chance to communicate with the RSU. We proposed to adapt $C W_{\min }$ according to node velocity, and determined optimal $C W_{\min }$ values required to achieve fairness (in the sense that all nodes with different speeds have same chance of communicating during their residence time in the coverage area of an $\mathrm{RSU})$. It was proved that these optimal $C W_{\min }$ values are independent of number of vehicles in the network. Analytical and simulation results were presented for the data transferred for vehicles belonging to different velocity classes. The impact of variability of vehicle speed were also analyzed. Adjusting TXOP rather than minimum CW, to achieve the desired fairness objective, is for the future study.

\section{Endnote}

${ }^{\mathrm{a}}$ We adopt the short notation: $P\left(z_{1}, s_{1}, b_{1} \mid z_{0}, s_{0}, b_{0}\right)=P$ $\left(z_{i}(t+1)=z_{1}, s_{i}(t+1)=s_{1}, b_{i}(t+1)=b_{1} \mid z_{i}(t)=z_{0}, s_{i}(t)=s_{0}\right.$, $\left.b_{i}(t)=b_{0}\right)$ 


\section{Competing interests}

The authors declare that they have no competing interests.

Received: 12 September 2011 Accepted: 14 May 2012

Published: 14 May 2012

\section{References}

1. S Yousefi, MS Mousavi, M Fathy, Vehicular ad hoc networks (VANETs): challenges and perspectives, in proc 6th IEEE Int Conf ITST, Chengdu, China, 761-766 (2006)

2. H Hartenstein, KP Laberteaux, A tutorial survey on vehicular ad hoc networks. IEEE Commun Mag. 46(6), 164-171 (2008)

3. R Uzcategui, G Acosta-Marum, Wave: a tutorial. IEEE Commun Mag. 47(5), 126-133 (2009)

4. IEEE P802.11 p/D5.0, draft amendment to standard for information technology telecommunications and information exchange between systems LAN/MAN specific requirements Part 11: WLAN medium access control (MAC) and physical layer (PHY) specifications: wireless access in vehicular environments (WAVE) (2008)

5. IEEE Standard for Information technology - Telecommunications and information exchange between systems - Local and metropolitan area networks - Specific requirements - Part 11: Wireless LAN MAC and PHY Specifications (2007)

6. IEEE 802.11e/D4.0, Draft Supplement to Part 11: Wireless LAN MAC and PHY specifications: MAC Enhancements for Quality of Service (QoS) (November 2005)

7. G Bianchi, Performance analysis of the IEEE 802.11 distributed coordination function. IEEE JSAC. 18(3), 535-547 (2000)

8. F Cali, M Conti, E Gregori, Dynamic tuning of the IEEE 802.11 protocol to achieve a theoretical throughput limit. IEEE/ACM Trans Network. 8(6), 785-799 (2000). doi:10.1109/90.893874

9. DJ Deng, $\mathrm{CH}$ Ke, HH Chen, YM Huang, Contention window optimization for IEEE 802.11 DCF access control. IEEE Trans Wirel Commun. 7(12), 5129-5135 (2008)

10. AV Babu, L Jacob, Fairness analysis of IEEE 802.11 multi-rate wireless LAN. IEEE Trans Veh Technol. 56, 3073-3088 (2007)

11. Y Xiao, Performance analysis of priority schemes for IEEE 802.11 and IEEE 802.11e wireless LANs. IEEE Trans Wirel Commun. 4(4), 1506-1515 (2005)

12. $X$ Chen, HH Refai, $X$ Ma, On the enhancements to IEEE 802.11 MAC and their suitability for safety-critical applications in VANET. Wirel Commun Mob Comput. 10(9), 1530-8677 (2008)

13. X Ma, X Chen, HH Refai, Performance and reliability of DSRC vehicular safety communication: a formal analysis. EURASIP J Wirel Commun Network. 2009, 13 (2009). Article ID 969164

14. Y Wang, A Ahmed, B Krishnamachari, K Psounis, IEEE 802.11p performance evaluation and protocol enhancement, in proc IEEE ICVES, Columbus Ohio, 317-322 (2008)

15. TH Luan, $X$ Ling, $X$ (Sherman) Shen, MAC performance analysis for vehicleto-infrastructure communication, in Proc Wireless Communications and Networking Conference (WCNC), Sydney, (1-6 April 2010)

16. J He, Z Tang, T O'Farrell, TM Chen, Performance analysis of DSRC priority mechanism for road safety applications in vehicular networks. Wirel Commun Mob Comput. 11(7), 980-990 (2009)

17. E Karamad, F Ashtiani, A modified 802.11-based MAC scheme to assure Fair Access for vehicle-to-roadside communications. Comput Commun. 31(12), 2898-2906 (2008). doi:10.1016/j.comcom.2008.01.030

18. S Eichler, Performance evaluation of the IEEE 802.11p WAVE communication standard, in Proc IEEE VTC-Fall, 2199-2203 (2007)

19. TH Luan, $X$ Ling, $X$ (Sherman) Shen, MAC in motion: impact of mobility on the MAC of drive-thru internet. IEEE Trans Mob Comput. 29(2), 305-319 (2012)

20. C Suthaputchakun, A Ganz, Secure priority based inter-vehicle communication MAC protocol for highway safety messaging, in 4th International Symposium on Wireless Communication Systems, Trondheim, 518-523 (2007)

21. Md Imrul Hassan, $\mathrm{HL} \mathrm{Vu}, \mathrm{T}$ Sakurai, Performance analysis of the IEEE 802.11 MAC protocol for DSRC with and without retransmissions, in IEEE International Symposium on a World of Wireless Mobile and Multimediab Networks, Montreal, QC, Canada, 1-8 (June 2010)
22. WL Tan, WC Lau, O Yue, Modeling resource sharing for a road-side access point supporting drive-thru internet, in Proc ACM VANET, New York, NY, USA, 33-42 (2009)

23. WL Tan, WC Lau, O Yue, TH Hui, Analytical models and performance evaluation of drive-thru internet systems. IEEE JSAC. 29(1), 207-222 (2011)

24. S-T Sheu, Y-C Cheng, J-S Wu, A channel access scheme to compromise throughput and fairness in IEEE $802.11 \mathrm{p}$ multi-rate/multi-channel wireless vehicular networks, in IEEE 71st Vehicular Technology Conference, Taipei, (1-5 May 20)

25. W Alasmary, W Zhuang, Mobility impact in IEEE 802.11 p infrastructure less vehicular networks. Ad Hoc Networks. 10(2), 222-230 (2010)

26. H Wu, RM Fujimoto, GF Riley, M Hunter, Spatial propagation of information in vehicular networks. IEEE Trans Veh Technol. 58(1), 420-431 (2009)

27. $R$ Jain, $W$ Hawe, $D$ Chiu, A quantitative measure of fairness and discrimination for resource allocation in shared computer systems. DEC Research Report TR-301 (September 1984)

28. RP Roess, ES Prassas, WR Mcshane, Traffic Engineering, 3rd edn. (Pearson Prentice Hall, Upper Saddle River, 2004)

doi:10.1186/1687-1499-2012-168

Cite this article as: Harigovindan et al.: Ensuring fair access in IEEE $802.11 \mathrm{p}$-based vehicle-to-infrastructure networks. EURASIP Journal on Wireless Communications and Networking 2012 2012:168.

\section{Submit your manuscript to a SpringerOpen ${ }^{\mathcal{O}}$ journal and benefit from:}

- Convenient online submission

- Rigorous peer review

- Immediate publication on acceptance

- Open access: articles freely available online

- High visibility within the field

- Retaining the copyright to your article

Submit your next manuscript at $\boldsymbol{~ s p r i n g e r o p e n . c o m ~}$ 